\title{
EXTREMAL GENERAL AFFINE SURFACE AREAS
}

\author{
STEVEN HOEHNER
}

\begin{abstract}
For a convex body $K$ in $\mathbb{R}^{n}$, we introduce and study the extremal general affine surface areas, defined by

$$
\operatorname{IS}_{\varphi}(K):=\sup _{K^{\prime} \subset K} \operatorname{as}_{\varphi}(K), \quad \operatorname{os}_{\psi}(K):=\inf _{K^{\prime} \supset K} \operatorname{as}_{\psi}(K)
$$

where $\operatorname{as}_{\varphi}(K)$ and $\operatorname{as}_{\psi}(K)$ are the $L_{\varphi}$ and $L_{\psi}$ affine surface area of $K$, respectively. We prove that there exist extremal convex bodies that achieve the supremum and infimum, and that the functionals $\mathrm{IS}_{\varphi}$ and $\mathrm{os}_{\psi}$ are continuous. In our main results, we prove Blaschke-Santaló type inequalities and inverse Santaló type inequalities for the extremal general affine surface areas. This article may be regarded as an Orlicz extension of the recent work of Giladi, Huang, Schütt and Werner (2020), who introduced and studied the extremal $L_{p}$ affine surface areas.
\end{abstract}

\section{INTRODUCTION}

The celebrated result of Fritz John [27] says that every convex body $K$ in $\mathbb{R}^{n}$ contains a unique ellipsoid of maximum volume, now called the John ellipsoid of $K$. The minimum volume ellipsoid containing $K$ is also unique and is called the Löwner ellipsoid of $K$. The John and Löwner ellipsoids are related by polar duality: If $K$ contains the origin in its interior, then the John ellipsoid is the polar body of the Löwner ellipsoid, and vice versa. These ellipsoids are cornerstones of asymptotic convex geometry, arising in concentration of volume, the reverse isoperimetric inequality, the hyperplane conjecture and many more [1, 11]. Other prominent applications of the John and Löwner ellipsoids can be found in Banach space geometry [10, 15, 44, 55], extremal problems [2, 7, 17, 48], polytopal approximation of convex bodies [20, 33, 52], linear programming [19, 56] and statistics [14, 45, 58].

In this article, we study an analogue of John's theorem when volume is replaced by the general $L_{\varphi}$ or $L_{\psi}$ affine surface area, which is defined in terms of a special type of concave function $\varphi$ or convex function $\psi$, respectively. More specifically, instead of considering the ellipsoid of maximum (resp. minimum) volume contained in (containing) $K$, we will consider the convex body of maximum $L_{\varphi}$ (minimum $L_{\psi}$ ) affine surface contained in (containing) $K$. When $K$ has centroid at the origin, we define the inner maximal $L_{\varphi}$ affine surface area $\operatorname{IS}_{\varphi}(K)$

Date: July 27, 2021.

2020 Mathematics Subject Classification. Primary: 52A20, 52A23, 52A40.

Key words and phrases. convex body, affine surface area, affine isoperimetric inequality, Blaschke-Santaló inequality, inverse Santaló inequality. 
and outer minimal $L_{\psi}$ affine surface area $\operatorname{os}_{\psi}(K)$ of $K$ by

$$
\begin{aligned}
\operatorname{IS}_{\varphi}(K) & :=\sup _{K^{\prime} \subset K} \operatorname{as}_{\varphi}\left(K^{\prime}\right) \\
\operatorname{Os}_{\psi}(K) & :=\inf _{K^{\prime} \supset K} \operatorname{as}_{\psi}\left(K^{\prime}\right) .
\end{aligned}
$$

Here $\operatorname{as}_{\varphi}(K)$ and $\operatorname{as}_{\psi}(K)$ denote the general $L_{\varphi}$ and $L_{\psi}$ affine surface area of $K$, respectively, which are extensions of the classical affine surface area defined by Schütt and Werner [51]. The supremum and infimum are taken over all $K^{\prime}$ with centroid at the origin (see Section 2.1 for the definitions).

The special case of (1) when $\mathrm{as}_{\varphi}$ is replaced by the classical affine surface area has attracted considerable interest. Inscribed bodies of maximal affine surface area have found applications to discrete geometry [3, 4, 5, 6], geometric probability [5] and variational problems in differential equations [32, 57]. The existence of a convex body $K_{\max } \subset K$ of maximum affine surface area follows from the upper semicontinuity of the affine surface area [4, 50]. The regularity properties of $K_{\max }$ were studied by Sheng, Trudinger and Wang [54]. For planar convex bodies, Bárány proved that $K_{\max }$ is unique, and showed a remarkable relationship between $K_{\max }$ and the limit shape of the convex hull of certain lattice points [4, 6] or random points [5] contained in $K$. Bárány and Prodromou [6] showed that if $K$ is a planar convex body, then $K_{\max }$ is of elliptic type, and if $K$ is of elliptic type then $K_{\max }=K$. Schneider [50] used a new method to extend the latter result to all dimensions, showing that if $K$ is a convex body in $\mathbb{R}^{n}$ of elliptic type, then $K_{\max }=K$.

Recently, Giladi, Huang, Schütt and Werner [18] introduced and studied the extremal $L_{p}$ affine surface areas

$$
\begin{aligned}
\operatorname{IS}_{p}(K) & :=\sup _{K^{\prime} \subset K} \operatorname{as}_{p}\left(K^{\prime}\right) \\
\operatorname{Os}_{p}(K) & :=\inf _{K^{\prime} \supset K} \operatorname{as}_{p}\left(K^{\prime}\right)
\end{aligned}
$$

where $\operatorname{as}_{p}(K)$ is the $L_{p}$ affine surface area of $K$. It was shown in 18 that for any convex body $K$ with centroid at the origin, the extremal bodies exist, and continuity and affine isoperimetric inequalities were proved for the functionals $\mathrm{IS}_{p}$ and $\mathrm{os}_{p}$. Asymptotic estimates were also given for $\operatorname{IS}_{p}(K)$ and $\operatorname{os}_{p}(K)$ in terms of powers of the volume of $K$ using the Löwner ellipsoid and thin shell estimate of [21].

When a certain concave or convex function depending on $n$ and $p$ is chosen in (11) or (2), respectively, we recover (3) and (44) as special cases. Thus, the new definitions (11) and (2) may be thought of as Orlicz extensions of the definitions (3) and (4) (see Section 6.4 below for the details). A key difference between the $L_{p}$ affine surface areas and the general $L_{\varphi}$ and $L_{\psi}$ affine surface areas is that the former are homogeneous, while the latter, in general, are not. For our purposes, the lack of homogeneity will only present difficulties for the $L_{\varphi}$ affine surface areas. To surmount this obstacle, we will restrict our attention to certain subclasses of concave functions $\varphi$ satisfying mild growth rate conditions. These subclasses contain many functions that are not homogeneous of any degree, which distinguishes the results in this 
work from those in [18. At the same time, these classes contain the homogeneous functions considered in the $L_{p}$ setting of [18].

In the present paper, we prove existence and continuity of the extremal general affine surface areas (11) and (2). Our main result is a Blaschke-Santaló type inequality for the inner maximal $L_{\varphi}$ affine surface area. More specifically, in Theorem 6.4 we show that for any convex body $K$ in $\mathbb{R}^{n}$ with centroid at the origin and any concave function $\varphi$ satisfying some prescribed conditions,

$$
\operatorname{IS}_{\varphi}(K) \operatorname{IS}_{\varphi}\left(K^{\circ}\right) \leq \operatorname{IS}_{\varphi}\left(B_{n}\right)^{2}
$$

with equality if and only if $K$ is an ellipsoid (here $B_{n}$ denotes the Euclidean unit ball $\mathbb{R}^{n}$ centered at the origin). Finally, we prove an inverse Santaló type inequality for the outer minimal $L_{\psi}$ affine surface area.

1.1. Overview. In Section 2, we state definitions and notation used throughout the paper, and provide the relevant background on the general $L_{\varphi}$ and $L_{\psi}$ affine surface areas. In Section 3. we use a polar duality relation of Ludwig [34] for the $L_{\varphi}$ affine surface areas to define the subclasses of concave functions $\varphi$ we will consider. Next, in Section 4 we define the extremal general affine surface areas and state their existence, monotonicity and continuity properties; for the reader's convenience, the proofs of these properties are included at the end of the paper in Section 7 . The lemmas that will be used in the proofs of the main results are in Section 5, and in Section 6 we prove affine isoperimetric inequalities for the extremal general affine surface areas. In Subsections 6.2 and 6.3 we prove our main results, which are BlaschkeSantaló type inequalities and inverse Santaló type inequalities for the extremal general affine surface areas.

\section{BACKGROUND AND NOTATION}

The standard inner product of $x, y \in \mathbb{R}^{n}$ is denoted $\langle x, y\rangle$. The Euclidean unit ball in $\mathbb{R}^{n}$ centered at the origin $o$ is the set $B_{n}=\left\{x \in \mathbb{R}^{n}:\langle x, x\rangle \leq 1\right\}$. The Minkowski sum $A+B$ of two sets $A, B \subset \mathbb{R}^{n}$ is defined by $A+B=\{a+b: a \in A, b \in B\}$. We say that a function $f:(0, \infty) \rightarrow(0, \infty)$ is homogeneous of degree $r$ if $f(\lambda t)=\lambda^{r} f(t)$ for all $\lambda, t>0$.

A convex body in $\mathbb{R}^{n}$ is a convex, compact set with nonempty interior. Denote the class of convex bodies in $\mathbb{R}^{n}$ that contain the origin in their interiors by $\mathcal{K}_{o}^{n}$. For convex bodies $K$ and $L$ in $\mathbb{R}^{n}$, the Hausdorff distance $\delta_{H}(K, L)$ of $K$ and $L$ is defined as

$$
\delta_{H}(K, L)=\inf \left\{\varepsilon \geq 0: K \subset L+\varepsilon B_{n}, L \subset K+\varepsilon B_{n}\right\} .
$$

The $n$-dimensional volume of $K$ is $|K|=\int_{\mathbb{R}^{n}} \mathbb{1}_{K}(x) d x$. The volume radius $\operatorname{vrad}(K)$ of $K$ is the radius of the Euclidean ball with the same volume as $K$, that is, $\operatorname{vrad}(K)=\left(|K| /\left|B_{n}\right|\right)^{1 / n}$. The centroid $g(K)$ of $K$ is defined by $g(K)=|K|^{-1} \int_{K} x d x$. If the centroid of $K$ is not the origin, then we translate $K$ so that it is. If $K \in \mathcal{K}_{o}^{n}$, then the polar body $K^{\circ}$ of $K$ is the convex body $K^{\circ}=\left\{x \in \mathbb{R}^{n}:\langle x, y\rangle \leq 1, \forall y \in K\right\}$. The bipolar theorem states that $\left(K^{\circ}\right)^{\circ}=K$. The polarity operation is inclusion-reversing, i.e., $L \subset K$ if and only if $L^{\circ} \supset K^{\circ}$. 
We also let $\partial K$ denote the boundary of $K$. In particular, the Euclidean unit ball has surface area $\left|\partial B_{n}\right|=n\left|B_{n}\right|$. The Gaussian curvature of $K$ at $x \in \partial K$ is denoted $\kappa(K, x)$, $\mu_{\partial K}$ is the usual surface measure on $\partial K$ and $N_{\partial K}(x)$ is the outer unit normal at $x \in \partial K$. For more background on convex geometry, we refer the reader to, e.g., the monograph of Schneider [49].

2.1. General $L_{\varphi}$ and $L_{\psi}$ affine surface areas. In the past decade or so, there has been tremendous interest in extending results from the $L_{p}$ theory of convex bodies to the rapidly growing Orlicz theory. Prominent examples include the Orlicz-Petty projection inequality [39] and Orlicz centroid inequalities [13, 31, 38, 67], the Orlicz-Brunn-Minkowski theory [16, 63], the Orlicz-Minkowski problem [22, 23, 26], Orlicz affine isoperimetric inequalities [12, 64, 65, 66] and Orlicz-John ellipsoids [68. The $L_{\varphi}$ and $L_{\psi}$ general affine surface areas, which are generalizations of the classical affine surface area, have played a key role in the initiation and development of this program.

The $L_{\varphi}$ affine surface area has its origins in valuation theory, arising in a fundamental result on the classification of upper semicontinuous $\operatorname{SL}(n)$ invariant valuations on $\mathcal{K}_{o}^{n}$. In the groundbreaking work [35], Ludwig and Reitzner proved that a functional $\Phi: \mathcal{K}_{o}^{n} \rightarrow \mathbb{R}$ is an upper semicontinuous and $\operatorname{SL}(n)$ invariant valuation that vanishes on polytopes in $\mathcal{K}_{o}^{n}$ if and only if there exists a concave function $\varphi:[0, \infty) \rightarrow[0, \infty)$ with $\lim _{t \rightarrow 0} \varphi(t)=\lim _{t \rightarrow \infty} \varphi(t) / t=$ 0 such that

$$
\Phi(K)=\int_{\partial K} \varphi\left(\frac{\kappa(K, x)}{\left\langle x, N_{\partial K}(x)\right\rangle^{n+1}}\right)\left\langle x, N_{\partial K}(x)\right\rangle d \mu_{\partial K}(x)
$$

for every $K \in \mathcal{K}_{o}^{n}$. The integral on the right-hand side of (6) is called the $L_{\varphi}$ affine surface area of $K$ and is denoted by $\operatorname{as}_{\varphi}(K)$. That is,

$$
\operatorname{as}_{\varphi}(K):=\int_{\partial K} \varphi\left(\frac{\kappa(K, x)}{\left\langle x, N_{\partial K}(x)\right\rangle^{n+1}}\right)\left\langle x, N_{\partial K}(x)\right\rangle d \mu_{\partial K}(x) .
$$

In view of this result, let $\operatorname{Conc}(0, \infty)$ denote the class of all concave functions $\varphi:(0, \infty) \rightarrow$ $(0, \infty)$ such that $\lim _{t \rightarrow 0} \varphi(t)=\lim _{t \rightarrow \infty} \frac{\varphi(t)}{t}=0$, and set $\varphi(0)=0$. (Examples of such functions include $\varphi(t)=t^{\alpha}$ for $\alpha \in(0,1), \varphi(t)=\log (t+1)$ and $\varphi(t)=\arctan (t)$.) When $\varphi(t)=t^{\frac{p}{n+p}}$ for $p>0$, one recovers the $L_{p}$ affine surface area; further choosing $p=1$ so that $\varphi(t)=t^{\frac{1}{n+1}}$, one obtains the classical affine surface area (see Subsection 6.4).

The $L_{\psi}$ affine surface area was introduced and studied in 34] as a generalization of the $L_{p}$ affine surface area for $p \in(-n, 0)$. Let $\operatorname{Conv}(0, \infty)$ denote the class of all convex functions $\psi:(0, \infty) \rightarrow(0, \infty)$ such that $\lim _{t \rightarrow 0} \psi(t)=\infty$ and $\lim _{t \rightarrow \infty} \psi(t)=0$, and set $\psi(0)=\infty$. (Examples of such functions include $\psi(t)=t^{\alpha}$ for $\alpha \in(-\infty, 0)$ and $\psi(t)=\log \left(t^{-1}+1\right)$.) For $K \in \mathcal{K}_{o}^{n}$ and $\psi \in \operatorname{Conv}(0, \infty)$, the $L_{\psi}$ general affine surface area $\operatorname{as}_{\psi}(K)$ is defined by [34]

$$
\operatorname{as}_{\psi}(K):=\int_{\partial K} \psi\left(\frac{\kappa(K, x)}{\left\langle x, N_{\partial K}(x)\right\rangle^{n+1}}\right)\left\langle x, N_{\partial K}(x)\right\rangle d \mu_{\partial K}(x) .
$$


Note that if $P \in \mathcal{K}_{o}^{n}$ is a polytope, then $\kappa(P, x)=0$ for almost all $x \in \partial P$, so $\operatorname{as}_{\psi}(P)=\infty$. When $\psi(t)=t^{\frac{p}{n+p}}$ and $p \in(-n, 0)$, one recovers the $L_{p}$ affine surface area (see Subsection 6.4).

As mentioned before, for $\varphi \in \operatorname{Conc}(0, \infty)$ the $L_{\varphi}$ affine surface area is upper semicontinuous, meaning for any sequence of convex bodies $\left\{K_{j}\right\}_{j \in \mathbb{N}} \subset \mathcal{K}_{o}^{n}$ that converges to $K$ with respect to the Hausdorff metric,

$$
\limsup _{j \rightarrow \infty} \operatorname{as}_{\varphi}\left(K_{j}\right) \leq \operatorname{as}_{\varphi}(K) .
$$

For $\psi \in \operatorname{Conv}(0, \infty)$, Ludwig 34 proved that the $L_{\psi}$ affine surface area is lower semicontinuous, meaning for any sequence of convex bodies $\left\{K_{j}\right\}_{j \in \mathbb{N}} \subset \mathcal{K}_{o}^{n}$ that converges to $K$ with respect to the Hausdorff metric,

$$
\liminf _{j \rightarrow \infty} \operatorname{as}_{\psi}\left(K_{j}\right) \geq \operatorname{as}_{\psi}(K) .
$$

The $L_{\psi}$ affine surface area is also $\operatorname{SL}(n)$ invariant [34].

The $L_{\varphi}$ and $L_{\psi}$ affine surface areas satisfy the following powerful affine isoperimetric inequalities due to Ludwig 34.

Lemma 2.1. [34, Thm. 3] Let $K$ be a convex body in $\mathbb{R}^{n}$ with centroid at the origin. Then for any $\varphi \in \operatorname{Conc}(0, \infty)$,

$$
\operatorname{as}_{\varphi}(K) \leq \operatorname{as}_{\varphi}\left(\operatorname{vrad}(K) B_{n}\right) .
$$

Moreover, there is equality for strictly increasing $\varphi$ if and only if $K$ is an ellipsoid.

Lemma 2.2. [34, Thm. 8] Let $K$ be a convex body in $\mathbb{R}^{n}$ with centroid at the origin. Then for any $\psi \in \operatorname{Conv}(0, \infty)$,

$$
\operatorname{as}_{\psi}(K) \geq \operatorname{as}_{\psi}\left(\operatorname{vrad}(K) B_{n}\right) .
$$

Moreover, there is equality for strictly decreasing $\psi$ if and only if $K$ is an ellipsoid.

\section{Polar DUAlity And the Functional Setting}

If $K=r B_{n}$ for some positive number $r$, then by definitions (17) and (8) we obtain

$$
\begin{aligned}
& \operatorname{as}_{\varphi}\left(r B_{n}\right)=r^{n} \varphi\left(r^{-2 n}\right)\left|\partial B_{n}\right| \\
& \operatorname{as}_{\psi}\left(r B_{n}\right)=r^{n} \psi\left(r^{-2 n}\right)\left|\partial B_{n}\right| .
\end{aligned}
$$

Since $\psi$ is decreasing, the $L_{\psi}$ affine surface areas are increasing on Euclidean balls. More specifically,

$$
0<r \leq s \quad \Longrightarrow \quad \operatorname{as}_{\psi}\left(r B_{n}\right) \leq \operatorname{as}_{\psi}\left(s B_{n}\right)
$$

This is not the case for the $L_{\varphi}$ affine surface area and a general function $\varphi \in \operatorname{Conc}(0, \infty)$. Fortunately, we can control the monotonicity of $\mathrm{as}_{\varphi}$ on Euclidean balls by restricting the growth rate of the ratio of $\varphi$ and the square root function. We will accomplish this via the 
following polar duality relation of Ludwig [34]. For $\varphi \in \operatorname{Conc}(0, \infty)$, define $\varphi^{*}(t):=t \varphi(1 / t)$. Then $\varphi^{*}$ is also an element of Conc $(0, \infty)$. Ludwig [34, Thm. 4] proved that for every $K \in \mathcal{K}_{o}^{n}$,

$$
\operatorname{as}_{\varphi}(K)=\operatorname{as}_{\varphi^{*}}\left(K^{\circ}\right)
$$

In this way, the operation $\varphi \mapsto \varphi^{*}$ induces a duality relationship within the class Conc $(0, \infty)$. Now define $\operatorname{Conc}^{-}(0, \infty)$ to be the class of all $\varphi \in \operatorname{Conc}(0, \infty)$ such that $\varphi(t) / \sqrt{t}$ is strictly decreasing, and define $\operatorname{Conc}^{+}(0, \infty)$ to be the class of all $\varphi \in \operatorname{Conc}(0, \infty)$ such that $\varphi(t) / \sqrt{t}$ is strictly increasing. The classes $\operatorname{Conc}^{-}(0, \infty)$ and $\operatorname{Conc}^{+}(0, \infty)$ are dual to one another in the following sense.

Lemma 3.1. $\varphi \in \operatorname{Conc}^{-}(0, \infty)$ if and only if $\varphi^{*} \in \operatorname{Conc}^{+}(0, \infty)$.

Proof. Let $\varphi \in \operatorname{Conc}^{-}(0, \infty), \varphi^{*} \in \operatorname{Conc}^{+}(0, \infty)$ and $t, u \in(0, \infty)$ with $t<u$. Also let $s=1 / t$ and $v=1 / u$ and note that $s>v$. Then

$$
\frac{\varphi(t)}{\sqrt{t}} \geq \frac{\varphi(u)}{\sqrt{u}} \Longleftrightarrow \frac{t \varphi^{*}(1 / t)}{\sqrt{t}} \geq \frac{u \varphi^{*}(1 / u)}{\sqrt{u}} \Longleftrightarrow \sqrt{t} \varphi^{*}(1 / t) \geq \sqrt{u} \varphi^{*}(1 / u) \Longleftrightarrow \frac{\varphi^{*}(s)}{\sqrt{s}} \geq \frac{\varphi^{*}(v)}{\sqrt{v}} .
$$

Note that if $\varphi(t)=\varphi(1) \sqrt{t}$, then $\varphi(t)=\varphi^{*}(t)$ and for any $r>0, \operatorname{as}_{\varphi}\left(r B_{n}\right)=\varphi(1)\left|\partial B_{n}\right|$ is constant with respect to $r$. Combined with Lemma 3.1, the next result shows that the "self-dual" function $\varphi(t)=\varphi(1) \sqrt{t}$ serves as a transition where the $L_{\varphi}$ affine surface area reverses monotonicity on Euclidean balls.

Lemma 3.2. Let $\varphi \in \mathrm{Conc}^{-}(0, \infty)$, and let $r, s>0$ with $r \leq s$. Then

$$
\begin{aligned}
\operatorname{as}_{\varphi}\left(r B_{n}\right) & \leq \operatorname{as}_{\varphi}\left(s B_{n}\right) \\
\operatorname{as}_{\varphi^{*}}\left(r B_{n}\right) & \geq \operatorname{as}_{\varphi^{*}}\left(s B_{n}\right) .
\end{aligned}
$$

Proof. Set $x=r^{-2 n}$ and $y=s^{-2 n}$. Then $x \geq y$, so by (11) and Lemma 3.1,

$$
\begin{gathered}
\operatorname{as}_{\varphi}\left(r B_{n}\right)=\frac{\varphi(x)}{\sqrt{x}}\left|\partial B_{n}\right| \leq \frac{\varphi(y)}{\sqrt{y}}\left|\partial B_{n}\right|=\operatorname{as}_{\varphi}\left(s B_{n}\right) \\
\operatorname{as}_{\varphi^{*}}\left(r B_{n}\right)=\frac{\varphi^{*}(x)}{\sqrt{x}}\left|\partial B_{n}\right| \geq \frac{\varphi^{*}(y)}{\sqrt{y}}\left|\partial B_{n}\right|=\operatorname{as}_{\varphi^{*}}\left(s B_{n}\right) .
\end{gathered}
$$

Remark 3.3. The family of functions $\varphi_{m}:(0, \infty) \rightarrow(0, \infty)$ defined by $\varphi_{m}(t):=\arctan \left(t^{1 / m}\right)$, $m \geq 2$, is a subclass of $\mathrm{Conc}^{-}(0, \infty)$; see Subsection 7.3 for the details. Hence by Lemma 3.1 the family $\varphi_{m}^{*}(t)=t \arctan \left(t^{-1 / m}\right), m \geq 2$, is a subclass of $\operatorname{Conc}^{+}(0, \infty)$. Note that, for any $r$, the function $\varphi_{m}$ is not homogeneous of degree $r$. To see this, observe that if a function $f$ is homogeneous of degree $r$, then $f(t)=f(1) t^{r}$ and hence $f(t) f(1 / t)=f(1)^{2}$ for all $t$. Thus if $\varphi_{m}$ is homogeneous for some $r$, then $\arctan \left(t^{1 / m}\right) \arctan \left(t^{-1 / m}\right)=\arctan (1)^{2}=\pi^{2} / 16$ for all $t$, a contradiction. 
Finally, we remark that the inclusions $\operatorname{Conc}^{-}(0, \infty) \subsetneq \operatorname{Conc}(0, \infty)$ and $\operatorname{Conc}^{+}(0, \infty) \subsetneq$ Conc $(0, \infty)$ are strict, as the example $\varphi(t)=\log (t+1)$ shows.

\section{Extremal General AFFine SURFACE AREAS}

For a convex body $K$ in $\mathbb{R}^{n}$ with $g(K)=o$, let

$$
\begin{aligned}
\mathscr{C}_{\text {in }}(K) & :=\left\{K^{\prime} \subset K: K^{\prime} \text { is a convex body in } \mathbb{R}^{n}, g\left(K^{\prime}\right)=o\right\} \\
\mathscr{C}_{\text {out }}(K) & :=\left\{K^{\prime} \supset K: K^{\prime} \text { is a convex body in } \mathbb{R}^{n}, g\left(K^{\prime}\right)=o\right\},
\end{aligned}
$$

denote the families of all inscribed and circumscribed bodies with centroid at the origin, respectively. The condition $g\left(K^{\prime}\right)=o$ allows us to apply Lemmas 2.1 and 2.2. A discussion on this centroid assumption can be found in Subsection [7.4. For $\varphi \in \operatorname{Conc}(0, \infty)$, we define the extremal $L_{\varphi}$ affine surface areas by

$$
\begin{aligned}
& \operatorname{IS}_{\varphi}(K):=\sup _{K^{\prime} \in \mathscr{C}_{\text {in }}(K)} \operatorname{as}_{\varphi}\left(K^{\prime}\right), \quad \operatorname{is}_{\varphi}(K):=\inf _{K^{\prime} \in \mathscr{C}_{\text {in }}(K)} \operatorname{as}_{\varphi}\left(K^{\prime}\right) \\
& \operatorname{OS}_{\varphi}(K):=\sup _{K^{\prime} \in \mathscr{C}_{\text {out }}(K)} \operatorname{as}_{\varphi}\left(K^{\prime}\right), \quad \operatorname{os}_{\varphi}(K):=\inf _{K^{\prime} \in \mathscr{C}_{\text {out }}(K)} \operatorname{as}_{\varphi}\left(K^{\prime}\right) .
\end{aligned}
$$

Likewise, for $\psi \in \operatorname{Conv}(0, \infty)$ we define the extremal $L_{\psi}$ affine surface areas by

$$
\begin{aligned}
& \operatorname{IS}_{\psi}(K):= \sup _{K^{\prime} \in \mathscr{C}_{\text {in }}(K)} \operatorname{as}_{\psi}\left(K^{\prime}\right), \quad \operatorname{is} \psi(K):=\inf _{K^{\prime} \in \mathscr{C}_{\text {in }}(K)} \operatorname{as}_{\psi}\left(K^{\prime}\right) \\
& \mathrm{OS}_{\psi}(K):=\sup _{K^{\prime} \in \mathscr{C}_{\text {out }}(K)} \operatorname{as}_{\psi}\left(K^{\prime}\right), \quad \mathrm{OS}_{\psi}(K):=\inf _{K^{\prime} \in \mathscr{C}_{\text {out }}(K)} \operatorname{as}_{\psi}\left(K^{\prime}\right) .
\end{aligned}
$$

These definitions were motivated by those in [18], where the extremal $L_{p}$ affine surface areas were introduced; choosing a specific function $\varphi$ or $\psi$ defined in terms of $n$ and $p$, we recover their definitions (see Subsection 6.4). We will be especially interested in the inner maximal $L_{\varphi}$ affine surface area $\operatorname{IS}_{\varphi}(K)$ and the outer minimal $L_{\psi}$ affine surface area $\operatorname{os}_{\psi}(K)$. In Lemma 4.1, we show that for any convex body $K \in \mathcal{K}_{o}^{n}$ with $g(K)=o$ and any $\varphi \in \mathrm{Conc}^{-}(0, \infty)$ and $\psi \in \operatorname{Conv}(0, \infty)$, there exist $K_{\max }^{\varphi} \in \mathscr{C}_{\text {in }}(K)$ and $K_{\min }^{\psi} \in \mathscr{C}_{\text {out }}(K)$ satisfying $\operatorname{as}_{\varphi}\left(K_{\max }^{\varphi}\right)=$ $\operatorname{IS}_{\varphi}(K)$ and $\operatorname{as}_{\psi}\left(K_{\min }^{\psi}\right)=\operatorname{os}_{\psi}(K)$.

We begin by ruling out the trivial cases in the definitions above. Since the $L_{\varphi}$ and $L_{\psi}$ affine surface areas have a " $0 / \infty$ property" on polytopes, one might expect the same for some of the extremal affine surface areas. Indeed, if $P \in \mathscr{C}_{\text {in }}(K)$ is a polytope, then $\operatorname{as}_{\varphi}(P)=0$ and $\operatorname{as}_{\psi}(P)=\infty$, so is ${ }_{\varphi}(K)=0$ and $\operatorname{IS}_{\psi}(K)=\infty$. Similarly, if $Q \in \mathscr{C}_{\text {out }}(K)$ is a polytope, then $\mathrm{OS}_{\varphi}(K)=0$ and $\mathrm{OS}_{\psi}(K)=\infty$. Moreover, by (12) and the definition of $\operatorname{Conv}(0, \infty)$ we obtain

$$
\operatorname{is}_{\psi}(K) \leq \inf _{r B_{n} \in \mathscr{C}_{\text {in }}(K)} \operatorname{as}_{\psi}\left(r B_{n}\right)=\inf _{r B_{n} \in \mathscr{C}_{\text {in }}(K)} r^{n} \psi\left(r^{-2 n}\right)\left|\partial B_{n}\right|=0
$$

Let $K \in \mathcal{K}_{o}^{n}$. Then $K^{\prime} \in \mathscr{C}_{\text {in }}(K)$ if and only if $\left(K^{\prime}\right)^{\circ} \in \mathscr{C}_{\text {out }}\left(K^{\circ}\right)$. Thus by (14),

$$
\operatorname{IS}_{\varphi}(K)=\sup _{K^{\prime} \in \mathscr{C}_{\text {in }}(K)} \operatorname{as}_{\varphi}\left(K^{\prime}\right)=\sup _{\left(K^{\prime}\right)^{\circ} \in \mathscr{C}_{\text {out }}\left(K^{\circ}\right)} \operatorname{as}_{\varphi^{*}}\left(\left(K^{\prime}\right)^{\circ}\right)=\operatorname{OS}_{\varphi^{*}}\left(K^{\circ}\right)
$$


provided the suprema exist. Note that if $\varphi \in \operatorname{Conc}^{-}(0, \infty)$ and $\lim _{t \rightarrow 0} \frac{\varphi(t)}{\sqrt{t}}=\infty$, then by Lemmas 3.1 and 3.2 .

$$
\operatorname{IS}_{\varphi^{*}}(K)=\sup _{K^{\prime} \in \mathscr{C}_{\text {in }}(K)} \operatorname{as}_{\varphi^{*}}\left(K^{\prime}\right) \geq \sup _{r B_{n} \in \mathscr{C}_{\text {in }}(K)} \operatorname{as}_{\varphi^{*}}\left(r B_{n}\right)=\sup _{r B_{n} \in \mathscr{C}_{\text {in }}(K)} \frac{\varphi^{*}\left(r^{-2 n}\right)}{r^{-n}}\left|\partial B_{n}\right|=\infty .
$$

Moreover, since

$$
\lim _{t \rightarrow \infty} \frac{\varphi^{*}(t)}{\sqrt{t}}=\lim _{t \rightarrow \infty} \sqrt{t} \varphi(1 / t)=\lim _{s \rightarrow 0} \frac{\varphi(s)}{\sqrt{s}},
$$

by Lemma 3.1 we have $\varphi \in \operatorname{Conc}^{-}(0, \infty)$ and $\lim _{t \rightarrow 0} \frac{\varphi(t)}{\sqrt{t}}=\infty$ if and only if $\varphi^{*} \in \operatorname{Conc}^{+}(0, \infty)$ and $\lim _{t \rightarrow \infty} \frac{\varphi^{*}(t)}{\sqrt{t}}=\infty$. For any such function $\varphi$, we have $\operatorname{OS}_{\varphi}(K)=\infty$.

4.1. Extremal $L_{\psi}^{*}$ affine surface areas. For $K \in \mathcal{K}_{o}^{n}$ and $\psi \in \operatorname{Conv}(0, \infty)$, Ludwig [34] defined the $L_{\psi}^{*}$ affine surface area by $\operatorname{as}_{\psi}^{*}(K):=\operatorname{as}_{\psi}\left(K^{\circ}\right)$. When $\psi(t)=t^{\frac{n}{n+p}}$ and $p<-n$, one recovers the $L_{p}$ affine surface area defined by Schütt and Werner [53]. This leads to the definition of the extremal $L_{\psi}^{*}$ affine surface areas:

$$
\begin{aligned}
& \operatorname{IS}_{\psi}^{*}(K):=\sup _{K^{\prime} \in \mathscr{C}_{\text {in }}(K)} \operatorname{as}_{\psi}^{*}\left(K^{\prime}\right), \quad \operatorname{is}_{\psi}^{*}(K):=\inf _{K^{\prime} \in \mathscr{C}_{\text {in }}(K)} \operatorname{as}_{\psi}^{*}\left(K^{\prime}\right) \\
& \operatorname{OS}_{\psi}^{*}(K):=\sup _{K^{\prime} \in \mathscr{C}_{\text {out }}(K)} \operatorname{as}_{\psi}^{*}\left(K^{\prime}\right), \quad \operatorname{os}_{\psi}^{*}(K):=\inf _{K^{\prime} \in \mathscr{C}_{\text {out }}(K)} \operatorname{as}_{\psi}^{*}\left(K^{\prime}\right) .
\end{aligned}
$$

Again, since $K^{\prime} \in \mathscr{C}_{\text {in }}(K)$ if and only if $\left(K^{\prime}\right)^{\circ} \in \mathscr{C}_{\text {out }}\left(K^{\circ}\right)$, we have

$$
\begin{gathered}
\mathrm{IS}_{\psi}^{*}(K)=\mathrm{OS}_{\psi}\left(K^{\circ}\right)=\infty \\
\mathrm{OS}_{\psi}^{*}(K)=\mathrm{IS}_{\psi}\left(K^{\circ}\right)=\infty \\
\operatorname{OS}_{\psi}^{*}(K)=\operatorname{is}_{\psi}\left(K^{\circ}\right)=0
\end{gathered}
$$

and

$$
\operatorname{is}_{\psi}^{*}(K)=\operatorname{os}_{\psi}\left(K^{\circ}\right) .
$$

Thus, from the $L_{\psi}^{*}$ family we shall only consider the inner minimal $L_{\psi}^{*}$ affine surface area is $\psi_{\psi}^{*}$.

4.2. Summary. We summarize these cases in the following tables. As we will see later, the functionals $\mathrm{IS}_{\varphi}$ and $\mathrm{OS}_{\varphi^{*}}$ are finite for all $\varphi \in \operatorname{Conc}^{-}(0, \infty)$, while $\mathrm{OS}_{\psi}$ and is ${ }_{\psi}^{*}$ are finite for all $\psi \in \operatorname{Conv}(0, \infty)$. First, let $\varphi \in \operatorname{Conc}^{-}(0, \infty)$ be such that $\lim _{t \rightarrow 0} \frac{\varphi(t)}{\sqrt{t}}=\infty$. Then we have:

\begin{tabular}{c|c|c|c|c|c}
$\operatorname{IS}_{\varphi}(K)$ & $\operatorname{IS}_{\varphi *}(K)$ & $\operatorname{is}_{\varphi}(K)$ & $\operatorname{OS}_{\varphi}(K)$ & $\operatorname{OS}_{\varphi^{*}}(K)$ & $\operatorname{OS}_{\varphi}(K)$ \\
\hline$<\infty$ & $\infty$ & 0 & $\infty$ & $<\infty$ & 0
\end{tabular}

Among these functionals, we shall only be interested in $\operatorname{IS}_{\varphi}$ and $\mathrm{OS}_{\varphi^{*}}$ for $\varphi \in \mathrm{Conc}^{-}(0, \infty)$. Meanwhile, for $\psi \in \operatorname{Conv}(0, \infty)$ we have:

$$
\begin{array}{c|c|c|c|c}
\mathrm{IS}_{\psi}(K) & \mathrm{is}_{\psi}(K) & \mathrm{OS}_{\psi}(K) & \mathrm{OS}_{\psi}(K) & \text { is }_{\psi}^{*}(K) \\
\hline \infty & 0 & \infty & <\infty & <\infty
\end{array}
$$

Among these functionals, we shall only be interested in $\mathrm{os}_{\psi}$ and is $\psi_{\psi}^{*}$. 
4.3. Existence of extremal bodies. In our first result, we show that the extremal general affine surface areas exist for all $\varphi \in \operatorname{Conc}^{-}(0, \infty)$ and all $\psi \in \operatorname{Conv}(0, \infty)$. An analogous result was proved for the extremal $L_{p}$ affine surface areas in [18, Lem. 3.2].

Lemma 4.1. Let $K$ be a convex body in $\mathbb{R}^{n}$ with centroid at the origin, and let $\varphi \in \operatorname{Conc}^{-}(0, \infty)$ and $\psi \in \operatorname{Conv}(0, \infty)$.

(i) There exists $K_{\max }^{\varphi} \in \mathscr{C}_{\text {in }}(K)$ such that $\operatorname{IS}_{\varphi}(K)=\operatorname{as}_{\varphi}\left(K_{\max }^{\varphi}\right)$.

(ii) There exists $K_{\max }^{\varphi^{*}} \in \mathscr{C}_{\text {out }}(K)$ such that $\operatorname{OS}_{\varphi^{*}}(K)=\operatorname{as}_{\varphi^{*}}\left(K_{\max }^{\varphi^{*}}\right)$.

(iii) There exists $K_{\text {min }}^{\psi} \in \mathscr{C}_{\text {out }}(K)$ such that $\operatorname{os}_{\psi}(K)=\operatorname{as}_{\psi}\left(K_{\min }^{\psi}\right)$.

(iv) There exists $K_{\min }^{\psi^{*}} \in \mathscr{C}_{\text {in }}(K)$ such that $\operatorname{is}_{\psi}^{*}(K)=\operatorname{as}_{\psi}\left(K_{\min }^{\psi^{*}}\right)$.

We postpone the proof until Section 7 .

4.4. Monotonicity and Continuity. Although the $L_{\varphi}$ and $L_{\psi}$ affine surface areas are not monotone in general, the corresponding extremal affine surface areas are. If $K, L \in \mathcal{K}_{o}^{n}$ and $K \subset L$, then $\mathscr{C}_{\text {in }}(K) \subset \mathscr{C}_{\text {in }}(L)$ implies

$$
\begin{aligned}
& \operatorname{IS}_{\varphi}(K)=\sup _{K^{\prime} \in \mathscr{C}_{\text {in }}(K)} \operatorname{as}_{\varphi}\left(K^{\prime}\right) \leq \sup _{L^{\prime} \in \mathscr{C}_{\text {in }}(L)} \operatorname{as}_{\varphi}\left(L^{\prime}\right)=\operatorname{IS}_{\varphi}(L), \\
& \operatorname{is}_{\psi}^{*}(K)=\inf _{K^{\prime} \in \mathscr{C}_{\text {in }}(K)} \operatorname{as}_{\psi}^{*}\left(K^{\prime}\right) \geq \inf _{L^{\prime} \in \mathscr{C}_{\text {in }}(L)} \operatorname{as}_{\psi}^{*}\left(L^{\prime}\right)=\operatorname{is}_{\psi}^{*}(L),
\end{aligned}
$$

while $\mathscr{C}_{\text {out }}(K) \supset \mathscr{C}_{\text {out }}(L)$ implies

$$
\begin{aligned}
\operatorname{OS}_{\varphi^{*}}(K) & =\sup _{K^{\prime} \in \mathscr{C}_{\text {out }}(K)} \operatorname{as}_{\varphi^{*}}\left(K^{\prime}\right) \geq \sup _{L^{\prime} \in \mathscr{C}_{\text {out }}(L)} \operatorname{as}_{\varphi^{*}}\left(L^{\prime}\right)=\mathrm{OS}_{\varphi^{*}}(L) \\
\operatorname{OS}_{\psi}(K) & =\inf _{K^{\prime} \in \mathscr{C}_{\text {out }}(K)} \operatorname{as}_{\psi}\left(K^{\prime}\right) \leq \inf _{L^{\prime} \in \mathscr{C}_{\text {out }}(L)} \operatorname{as}_{\psi}\left(L^{\prime}\right)=\operatorname{os}_{\psi}(L) .
\end{aligned}
$$

The next result shows that the extremal general affine surface areas are continuous with respect to the Hausdorff metric (c.f. [18, Prop. 3.3]). This is not the case for the general $L_{\varphi}$ and $L_{\psi}$ affine surface areas, which are zero or infinite, respectively, on any sequence of polytopes that converges to a given convex body in the Hausdorff metric.

Proposition 4.2. Consider the class of convex bodies in $\mathbb{R}^{n}$ with centroid at the origin, equipped with the Hausdorff metric.

(i) For any $\varphi \in \operatorname{Conc}^{-}(0, \infty)$, the functional $K \mapsto \operatorname{IS}_{\varphi}(K)$ is continuous.

(ii) For any $\varphi^{*} \in \operatorname{Conc}^{+}(0, \infty)$, the functional $K \mapsto \mathrm{OS}_{\varphi^{*}}(K)$ is continuous.

(iii) For any $\psi \in \operatorname{Conv}(0, \infty)$, the functional $K \mapsto \mathrm{os}_{\psi}(K)$ is continuous.

(iv) For any $\psi \in \operatorname{Conv}(0, \infty)$, the functional $K \mapsto \operatorname{is}_{\psi}^{*}(K)$ is continuous.

The proof of Proposition 4.2 is also given in Section 7.

\section{LEMMAS}

The next lemma is elementary. We include the proof for the reader's convenience. 
Lemma 5.1. For all $\varphi \in \operatorname{Conc}^{-}(0, \infty), \varphi^{*} \in \operatorname{Conc}^{+}(0, \infty)$ and $\psi \in \operatorname{Conv}(0, \infty)$, we have:

$$
\begin{aligned}
\operatorname{IS}_{\varphi}\left(B_{n}\right) & =\operatorname{as}_{\varphi}\left(B_{n}\right)=\varphi(1)\left|\partial B_{n}\right| \\
\operatorname{OS}_{\varphi^{*}}\left(B_{n}\right) & =\operatorname{as}_{\varphi^{*}}\left(B_{n}\right)=\varphi(1)\left|\partial B_{n}\right| \\
\operatorname{OS}_{\psi}\left(B_{n}\right) & =\operatorname{as}_{\psi}\left(B_{n}\right)=\psi(1)\left|\partial B_{n}\right| \\
\operatorname{is}_{\psi}^{*}\left(B_{n}\right) & =\operatorname{as}_{\psi}^{*}\left(B_{n}\right)=\psi(1)\left|\partial B_{n}\right| .
\end{aligned}
$$

Proof. On one hand, we have $\operatorname{IS}_{\varphi}\left(B_{n}\right)=\sup _{K \in \mathscr{C}_{\text {in }}\left(B_{n}\right)} \operatorname{as}_{\varphi}(K) \geq \operatorname{as}_{\varphi}\left(B_{n}\right)$ since $B_{n} \in \mathscr{C}_{\text {in }}\left(B_{n}\right)$. On the other hand, by Lemmas 2.1 and 3.2 we derive

$$
\operatorname{IS}_{\varphi}\left(B_{n}\right)=\sup _{K \in \mathscr{C}_{\text {in }}\left(B_{n}\right)} \operatorname{as}_{\varphi}(K) \leq \sup _{K \in \mathscr{C}_{\text {in }}\left(B_{n}\right)} \operatorname{as}_{\varphi}\left(\operatorname{vrad}(K) B_{n}\right)=\operatorname{as}_{\varphi}\left(B_{n}\right) .
$$

Thus $\operatorname{IS}_{\varphi}\left(B_{n}\right)=\operatorname{as}_{\varphi}\left(B_{n}\right)$. By (17), we have $\operatorname{OS}_{\varphi^{*}}\left(B_{n}\right)=\operatorname{IS}_{\varphi}\left(B_{n}^{\circ}\right)=\operatorname{IS}_{\varphi}\left(B_{n}\right)$ since $B_{n}^{\circ}=B_{n}$. The argument to prove $\operatorname{os}_{\psi}\left(B_{n}\right)=\operatorname{as}_{\psi}\left(B_{n}\right)$ is similar, and by (18) we have is ${ }_{\psi}^{*}\left(B_{n}\right)=\operatorname{as}_{\psi}^{*}\left(B_{n}\right)$.

The identity $\operatorname{as}_{\varphi}\left(B_{n}\right)=\varphi(1)\left|\partial B_{n}\right|$ follows from (11), and $\operatorname{as}_{\psi}\left(B_{n}\right)=\psi(1)\left|\partial B_{n}\right|$ follows from (12). Finally, note that $\operatorname{as}_{\varphi}\left(B_{n}\right)=\operatorname{as}_{\varphi^{*}}\left(B_{n}\right)$ and $\operatorname{as}_{\psi}^{*}\left(B_{n}\right)=\operatorname{as}_{\psi}\left(B_{n}\right)$.

Another ingredient we will need describes the effect a dilation has on the general affine surface area of a convex body.

Lemma 5.2. Let $K \in \mathcal{K}_{o}^{n}, \varphi \in \operatorname{Conc}(0, \infty)$ and $\psi \in \operatorname{Conv}(0, \infty)$. Then for any $r \in[0,1]$ and any $R \geq 1$, the following inequalities hold:

(i) $\operatorname{as}_{\varphi}(r K) \geq r^{n} \operatorname{as}_{\varphi}(K)$

(ii) $\operatorname{as}_{\varphi}(R K) \leq R^{n} \operatorname{as}_{\varphi}(K)$

(iii) $\operatorname{as}_{\psi}(r K) \leq r^{n} \operatorname{as}_{\psi}(K)$

(iv) $\operatorname{as}_{\psi}(R K) \geq R^{n} \operatorname{as}_{\psi}(K)$.

Proof. We shall prove part (iv); the other cases (i)-(iii) proceed similarly. By the definition (8) of the $L_{\psi}$ affine surface area,

$$
\begin{aligned}
\operatorname{as}_{\psi}(r K) & =\int_{\partial r K} \psi\left(\frac{\kappa(r K, x)}{\left\langle x, N_{\partial r K}(x)\right\rangle^{n+1}}\right)\left\langle x, N_{\partial r K}(x)\right\rangle d \mu_{\partial r K}(x) \\
& =\int_{\partial r K} \psi\left(\frac{\kappa(r K, r y)}{\left\langle r y, N_{\partial r K}(r y)\right\rangle^{n+1}}\right)\left\langle r y, N_{\partial r K}(r y)\right\rangle d \mu_{\partial r K}(r y) \\
& =r^{n} \int_{\partial K} \psi\left(\frac{\kappa(K, y)}{r^{2 n}\left\langle y, N_{\partial K}(y)\right\rangle^{n+1}}\right)\left\langle y, N_{\partial K}(y)\right\rangle d \mu_{\partial K}(y) \\
& \leq r^{n} \int_{\partial K} \psi\left(\frac{\kappa(K, y)}{\left\langle y, N_{\partial K}(y)\right\rangle^{n+1}}\right)\left\langle y, N_{\partial K}(y)\right\rangle d \mu_{\partial K}(y) \\
& =r^{n} \operatorname{as}_{\psi}(K) .
\end{aligned}
$$

In the inequality we used the fact that $r \leq 1$ and $\psi$ is decreasing. 


\section{AfFine isoperimetric Inequalities}

Affine isoperimetric inequalities are among the most powerful tools in convex geometry. They relate two functionals on the class of convex bodies in $\mathbb{R}^{n}$, where the ratio of the functionals is invariant under nondegenerate linear transformations. Prominent examples include the classical affine isoperimetric inequality [30, 36, 24] and the Blaschke-Santaló inequality [8, 43, 46]. More recent examples include affine isoperimetric inequalities for the $L_{p}$ affine surface area [37, 40, 61, the mixed $L_{p}$ affine surface area [37, 59, 62, the general affine surface area [34, 65], the mixed general affine surface area [64] and the extremal $L_{p}$ affine surface areas [18. In our next two results, we provide affine isoperimetric inequalities for the extremal general affine surface areas.

Proposition 6.1. Let $K$ be a convex body in $\mathbb{R}^{n}$ with centroid at the origin. If $\varphi \in \mathrm{Conc}^{-}(0, \infty)$, then

$$
\begin{gathered}
\operatorname{IS}_{\varphi}(K) \leq \operatorname{vrad}(K)^{n} \cdot \frac{\varphi\left(\operatorname{vrad}(K)^{-2 n}\right)}{\varphi(1)} \cdot \operatorname{IS}_{\varphi}\left(B_{n}\right) \\
\operatorname{OS}_{\varphi^{*}}(K) \leq \operatorname{vrad}\left(K^{\circ}\right)^{-n} \cdot \frac{\varphi^{*}\left(\operatorname{vrad}\left(K^{\circ}\right)^{2 n}\right)}{\varphi^{*}(1)} \cdot \operatorname{OS}_{\varphi^{*}}\left(B_{n}\right) .
\end{gathered}
$$

Moreover, if $\varphi$ is strictly increasing, then equality holds in each case if and only if $K$ is an ellipsoid.

Proposition 6.2. Let $K$ be a convex body in $\mathbb{R}^{n}$ with centroid at the origin. If $\psi \in \operatorname{Conv}(0, \infty)$, then

$$
\begin{aligned}
& \operatorname{os}_{\psi}(K) \geq \operatorname{vrad}(K)^{n} \cdot \frac{\psi\left(\operatorname{vrad}(K)^{-2 n}\right)}{\psi(1)} \cdot \operatorname{os}_{\psi}\left(B_{n}\right) \\
& \operatorname{is}_{\psi}^{*}(K) \geq \operatorname{vrad}\left(K^{\circ}\right)^{n} \cdot \frac{\psi\left(\operatorname{vrad}\left(K^{\circ}\right)^{-2 n}\right)}{\psi(1)} \cdot \operatorname{is}_{\psi}^{*}\left(B_{n}\right) .
\end{aligned}
$$

Moreover, if $\psi$ is strictly decreasing, then equality holds in each case if and only if $K$ is an ellipsoid.

6.1. Proof of Propositions 6.1 and 6.2. By Lemmas 2.1, 3.2 and 5.1 we obtain

$$
\begin{aligned}
\operatorname{IS}_{\varphi}(K)=\sup _{K^{\prime} \in \mathscr{C}_{\text {in }}(K)} \operatorname{as}_{\varphi}\left(K^{\prime}\right) & \leq \sup _{K^{\prime} \in \mathscr{C}_{\text {in }}(K)} \operatorname{as}_{\varphi}\left(\operatorname{vrad}\left(K^{\prime}\right) B_{n}\right) \\
& =\operatorname{as}_{\varphi}\left(\operatorname{vrad}(K) B_{n}\right) \\
& =\operatorname{vrad}(K)^{n} \cdot \frac{\varphi\left(\operatorname{vrad}(K)^{-2 n}\right)}{\varphi(1)} \cdot \varphi(1)\left|\partial B_{n}\right| \\
& =\operatorname{vrad}(K)^{n} \cdot \frac{\varphi\left(\operatorname{vrad}(K)^{-2 n}\right)}{\varphi(1)} \cdot \operatorname{IS}_{\varphi}\left(B_{n}\right) .
\end{aligned}
$$


Next, we use (27), (17) and $\varphi^{*}(t)=t \varphi(1 / t)$ to get

$$
\begin{aligned}
\operatorname{OS}_{\varphi^{*}}(K)=\operatorname{IS}_{\varphi}\left(K^{\circ}\right) & \leq \operatorname{vrad}\left(K^{\circ}\right)^{n} \cdot \frac{\varphi\left(\operatorname{vrad}\left(K^{\circ}\right)^{-2 n}\right)}{\varphi(1)} \cdot \operatorname{OS}_{\varphi^{*}}\left(B_{n}\right) \\
& =\operatorname{vrad}\left(K^{\circ}\right)^{-n} \cdot \frac{\varphi^{*}\left(\operatorname{vrad}\left(K^{\circ}\right)^{2 n}\right)}{\varphi^{*}(1)} \cdot \operatorname{OS}_{\varphi^{*}}\left(B_{n}\right) .
\end{aligned}
$$

Similarly, using Lemma 2.2, Lemma 5.1 and (13) we derive

$$
\begin{aligned}
\operatorname{os}_{\psi}(K)=\inf _{K^{\prime} \in \mathscr{C}_{\text {out }}(K)} \operatorname{as}_{\psi}\left(K^{\prime}\right) & \geq \operatorname{as}_{\psi}\left(\operatorname{vrad}(K) B_{n}\right) \\
& =\operatorname{vrad}(K)^{n} \cdot \frac{\psi\left(\operatorname{vrad}(K)^{-2 n}\right)}{\psi(1)} \cdot \operatorname{os}_{\psi}\left(B_{n}\right) .
\end{aligned}
$$

From (29) and (18) we get (30). The equality conditions in each case follow from those in Lemmas 2.1 and 2.2 .

Remark 6.3. Choosing $\varphi(t)=t^{\frac{p}{n+p}}$ with $p \in[0, n]$ or $p \in[n, \infty]$ in Proposition 6.1] or $\psi(t)=t^{\frac{p}{n+p}}$ with $p \in(-n, 0]$ in Proposition 6.2. we recover the affine isoperimetric inequalities of Giladi, Huang, Schütt and Werner [18, Prop. 3.4] for the extremal $L_{p}$ affine surface areas.

6.2. Blaschke-Santaló type inequalities. The celebrated Blaschke-Santaló inequality states that for any convex body $K$ in $\mathbb{R}^{n}$ with centroid at the origin,

$$
|K| \cdot\left|K^{\circ}\right| \leq\left|B_{n}\right|^{2}
$$

with equality if and only if $K$ is an ellipsoid. For $n=2,3$, this result is due to Blaschke [8], and it was extended to all $n$ by Santaló [46]. The equality conditions were later proved by Petty [43. In particular, Blaschke-Santaló type inequalities have also been shown for the $L_{p}$ affine surface area and the mixed $L_{p}$ affine surface area (see [37, 59, 61, 62, 64] and the references therein), as well as to the general affine surface area [34] and mixed general affine surface area [64]. In our main result, we prove an analogue for the extremal general affine surface areas.

Theorem 6.4. Let $K$ be a convex body in $\mathbb{R}^{n}$ with centroid at the origin, and let $\varphi \in$ Conc $^{-}(0, \infty)$ satisfy the submultiplicativity condition $\varphi(t) \varphi(1 / t) \leq \varphi(1)^{2}$ for all $t>0$. Then

$$
\begin{aligned}
\operatorname{IS}_{\varphi}(K) \operatorname{IS}_{\varphi}\left(K^{\circ}\right) & \leq \operatorname{IS}_{\varphi}\left(B_{n}\right)^{2} \\
\operatorname{OS}_{\varphi^{*}}(K) \operatorname{OS}_{\varphi^{*}}\left(K^{\circ}\right) & \leq \operatorname{OS}_{\varphi^{*}}\left(B_{n}\right)^{2} .
\end{aligned}
$$

Moreover, if $\varphi$ is strictly increasing, then equality holds in each case if and only if $K$ is an ellipsoid.

Proof. Applying Proposition 6.1 to $K$ and $K^{\circ}$, we derive

$$
\operatorname{IS}_{\varphi}(K) \operatorname{IS}_{\varphi}\left(K^{\circ}\right) \leq \frac{\varphi\left(\operatorname{vrad}(K)^{-2 n}\right)}{\operatorname{vrad}(K)^{-n}} \cdot \frac{\varphi\left(\operatorname{vrad}\left(K^{\circ}\right)^{-2 n}\right)}{\operatorname{vrad}\left(K^{\circ}\right)^{-n}} \cdot \varphi(1)^{-2} \cdot \operatorname{IS}_{\varphi}\left(B_{n}\right)^{2} .
$$


It suffices to show that for all $s, t>0$ with $s t \geq 1$,

$$
\frac{\varphi(s)}{\sqrt{s}} \cdot \frac{\varphi(t)}{\sqrt{t}} \leq \varphi(1)^{2}
$$

for then the conclusion follows with $s=\operatorname{vrad}(K)^{-2 n}$ and $t=\operatorname{vrad}\left(K^{\circ}\right)^{-2 n}$, where st $\geq 1$ holds by the Blaschke-Santaló inequality. The function $t \mapsto \varphi(t) / \sqrt{t}$ is decreasing since $\varphi \in \operatorname{Conc}^{-}(0, \infty)$, so for all $s, t>0$ with $s \geq 1 / t$

$$
\frac{\varphi(s)}{\sqrt{s}} \cdot \frac{\varphi(t)}{\sqrt{t}} \leq \frac{\varphi(1 / t)}{\sqrt{1 / t}} \cdot \frac{\varphi(t)}{\sqrt{t}}=\varphi(t) \varphi(1 / t) .
$$

By hypothesis, this is less than or equal to $\varphi(1)^{2}$ for all $t>0$, which shows (34). Hence, inequality (31) follows from (33).

The identity $\operatorname{IS}_{\varphi}(K) \operatorname{IS}_{\varphi}\left(K^{\circ}\right)=\operatorname{OS}_{\varphi^{*}}(K) \operatorname{OS}_{\varphi^{*}}\left(K^{\circ}\right)$ follows from (17), and $\operatorname{IS}_{\varphi}\left(B_{n}\right)=\mathrm{OS}_{\varphi^{*}}\left(B_{n}\right)$ by Lemma 5.1. Thus, (32) follows from (31). The equality conditions follow from those in Proposition 6.1, since they are stronger than those of the Blaschke-Santaló inequality.

Remark 6.5. For any $m \geq 2$, the function $\varphi_{m}(t)=\arctan \left(t^{1 / m}\right)$ lies in $\operatorname{Conc}^{-}(0, \infty)$ by Remark 3.3. We show that it satisfies the submultiplicativity condition $\varphi_{m}(t) \varphi_{m}(1 / t) \leq$ $\varphi_{m}(1)^{2}$ for all $t>0$. This is equivalent to

$$
\arctan \left(t^{1 / m}\right) \arctan \left(t^{-1 / m}\right) \leq \frac{\pi^{2}}{16}, \quad \forall t>0 .
$$

We use the identity $\arctan (1 / x)=\pi / 2-\arctan (x)$ for all $x>0$ to derive

$$
\varphi_{m}(t) \varphi_{m}(1 / t)=\varphi_{m}(t)\left(\frac{\pi}{2}-\varphi_{m}(t)\right)=-\varphi_{m}(t)^{2}+\frac{\pi}{2} \cdot \varphi_{m}(t)
$$

Set $s=\varphi_{m}(t)$. The maximum value of the function $s \mapsto-s^{2}+\frac{\pi}{2} s$ is achieved when $s=\pi / 4$, which implies $t=\tan (\pi / 4)=1$. Therefore,

$$
\varphi_{m}(t) \varphi_{m}(1 / t) \leq-\varphi_{m}(1)^{2}+\frac{\pi}{2} \varphi_{m}(1)=\frac{\pi^{2}}{16},
$$

which proves (35). Thus, for $m \geq 2$ the function $\varphi_{m}$ satisfies the hypotheses of Theorem 6.4.

6.3. Inverse Santaló type inequalities. Bourgain and Milman's inverse Santaló inequality [10] (see also [28, 41, 42]) states that there exists an absolute constant $c>0$ such that for all $n \geq 1$ and every convex body $K$ in $\mathbb{R}^{n}$,

$$
|K| \cdot\left|K^{\circ}\right| \geq c^{n}\left|B_{n}\right|^{2} .
$$

The best known constant is $c=1 / 2$, which is due to Kuperberg [28].

Our next result gives inverse Santaló type inequalities for the outer minimal $L_{\psi}$ and inner minimal $L_{\psi}^{*}$ affine surface areas. 
Theorem 6.6. Let $K$ be a convex body in $\mathbb{R}^{n}$ with centroid at the origin. If $\psi \in \operatorname{Conv}(0, \infty)$, then there exists a positive absolute constant $c$ such that

$$
\begin{gathered}
\operatorname{os}_{\psi}(K) \operatorname{os}_{\psi}\left(K^{\circ}\right) \geq c^{n} \cdot \frac{\psi\left(\operatorname{vrad}(K)^{-2 n}\right) \psi\left(\operatorname{vrad}\left(K^{\circ}\right)^{-2 n}\right)}{\psi(1)^{2}} \cdot \operatorname{os}_{\psi}\left(B_{n}\right)^{2} \\
\operatorname{is}_{\psi}^{*}(K) \operatorname{is}_{\psi}^{*}\left(K^{\circ}\right) \geq c^{n} \cdot \frac{\psi\left(\operatorname{vrad}(K)^{-2 n}\right) \psi\left(\operatorname{vrad}\left(K^{\circ}\right)^{-2 n}\right)}{\psi(1)^{2}} \cdot \operatorname{is}_{\psi}^{*}\left(B_{n}\right)^{2} .
\end{gathered}
$$

Proof. By Proposition 6.2 and the inverse Santaló inequality (36),

$$
\begin{aligned}
\operatorname{os}_{\psi}(K) \operatorname{os}_{\psi}\left(K^{\circ}\right) & \geq\left(\operatorname{vrad}(K) \operatorname{vrad}\left(K^{\circ}\right)\right)^{n} \psi\left(\operatorname{vrad}(K)^{-2 n}\right) \psi\left(\operatorname{vrad}\left(K^{\circ}\right)^{-2 n}\right) \operatorname{os}_{\psi}\left(B_{n}\right)^{2} \\
& \geq c^{n} \cdot \frac{\psi\left(\operatorname{vrad}(K)^{-2 n}\right) \psi\left(\operatorname{vrad}\left(K^{\circ}\right)^{-2 n}\right)}{\psi(1)^{2}} \cdot \operatorname{os}_{\psi}\left(B_{n}\right)^{2} .
\end{aligned}
$$

Inequality (38) now follows from (18) and (26).

6.4. Extremal $L_{p}$ affine surface areas. Let $K$ be a convex body in $\mathbb{R}^{n}$ with centroid at the origin. For any real number $p \neq-n$, the $L_{p}$ affine surface area $\operatorname{as}_{p}(K)$ of $K$ is defined by

$$
\operatorname{as}_{p}(K)=\int_{\partial K} \frac{\kappa(K, x)^{\frac{p}{n+p}}}{\left\langle x, N_{K}(x)\right\rangle^{\frac{n(p-1)}{n+p}}} d \mu_{\partial K}(x)
$$

and [37, 53

$$
\operatorname{as}_{ \pm \infty}(K)=\int_{\partial K} \frac{\kappa(K, x)}{\left\langle x, N_{K}(x)\right\rangle^{n}} d \mu_{\partial K}(x),
$$

provided the integrals exist. These definitions were given in [37] for $p>1$ and [53] for $p<1$.

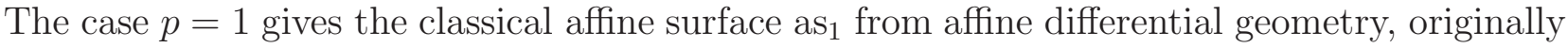
due to Blaschke 9 for sufficiently smooth convex bodies. The definition of as 1 was extended to all convex bodies in $\mathbb{R}^{n}$ by several authors [29, 36, 40, 47, 51, 60, with Schütt and Werner [51] showing specifically that the definition $\operatorname{as}_{1}(K)=\int_{\partial K} \kappa(K, x)^{\frac{1}{n+1}} d \mu_{\partial K}(x)$ extends naturally to all convex bodies in $\mathbb{R}^{n}$.

The $L_{p}$ affine surface area is homogeneous of degree $\frac{n(n-p)}{n+p}$ (see [53, Prop. 9]), meaning

$$
\operatorname{as}_{p}(\lambda K)=\lambda^{\frac{n(n-p)}{n+p}} \operatorname{as}_{p}\left(B_{n}\right), \quad \forall \lambda>0 .
$$

The following $L_{p}$ affine isoperimetric inequalities are due to Lutwak [37 for $p \geq 1$ and to Werner and Ye [61, Thm. 4.2] for $p<1$. For any convex body $K$ in $\mathbb{R}^{n}$ with centroid at the origin and all $p \geq 0$,

$$
\operatorname{as}_{p}(K) \leq \operatorname{vrad}(K)^{\frac{n(n-p)}{n+p}} \operatorname{as}_{p}\left(B_{n}\right)
$$

while for $-n<p \leq 0$ the inequality reverses,

$$
\operatorname{as}_{p}(K) \geq \operatorname{vrad}(K)^{\frac{n(n-p)}{n+p}} \operatorname{as}_{p}\left(B_{n}\right) .
$$


Equality holds in each case if and only if $K$ is an ellipsoid, and it holds trivially if $p=0$. For $p= \pm \infty$, it follows from [37, Thm. 7.7] and [53, pp. 114-115] that for any convex body $K$ in $\mathbb{R}^{n}$,

$$
\operatorname{as}_{ \pm \infty}(K) \leq n\left|K^{\circ}\right|
$$

with equality if and only if $K$ is an ellipsoid.

Giladi, Huang, Schütt and Werner [18] defined the inner and outer maximal $L_{p}$ affine surface areas by

$$
\operatorname{IS}_{p}(K)=\sup _{K^{\prime} \in \mathscr{C}_{\text {in }}(K)} \operatorname{as}_{p}\left(K^{\prime}\right), \quad \operatorname{OS}_{p}(K)=\sup _{K^{\prime} \in \mathscr{C}_{\text {out }}(K)} \operatorname{as}_{p}\left(K^{\prime}\right),
$$

respectively, and the inner and outer minimal $L_{p}$ affine surface areas by

$$
\operatorname{is}_{p}(K)=\inf _{K^{\prime} \in \mathscr{C}_{\text {in }}(K)} \operatorname{as}_{p}\left(K^{\prime}\right), \quad \operatorname{os}_{p}(K)=\inf _{K^{\prime} \in \mathscr{C}_{\text {out }}(K)} \operatorname{as}_{p}\left(K^{\prime}\right),
$$

respectively. The relevant $p$ ranges for $\mathrm{IS}_{p}, \mathrm{OS}_{p}$ and $\mathrm{os}_{p}$ are the intervals $[0, n],[n, \infty]$ and $(-n, 0]$ respectively; there is no interesting $p$ range for the functional is ${ }_{p}$, which is identically zero for all $K$ and all $p$. Taking $\varphi(t)=t^{\frac{p}{n+p}}$ or $\psi(t)=t^{\frac{p}{n+p}}$ with the corresponding $p$ interval, we recover these definitions from those of the extremal $L_{\varphi}$ and $L_{\psi}$ affine surface areas given in Section 4 .

As a corollary to Theorem 6.4, we obtain Blaschke-Santaló type inequalities for the extremal $L_{p}$ affine surface areas.

Corollary 6.7. Let $K$ be a convex body in $\mathbb{R}^{n}$ with centroid at the origin.

(i) For all $p \in[0, n]$ we have $\operatorname{IS}_{p}(K) \operatorname{IS}_{p}\left(K^{\circ}\right) \leq \operatorname{IS}_{p}\left(B_{n}\right)^{2}$. Equality holds trivially if $p=n$.

(ii) For all $p \in[n, \infty]$ we have $\operatorname{OS}_{p}(K) \mathrm{OS}_{p}\left(K^{\circ}\right) \leq \operatorname{OS}_{p}\left(B_{n}\right)^{2}$. Equality holds trivially if $p=n$.

Equality holds in each case if and only if $K$ is an ellipsoid.

Proof. Let $\varphi(t)=t^{\frac{p}{n+p}}$. For $p \in[0, n]$, we have $\frac{p}{n+p} \in[0,1 / 2]$. Thus, $\lim _{t \rightarrow 0} \varphi(t)=$ $\lim _{t \rightarrow \infty} \frac{\varphi(t)}{t}=0$ and $\varphi(0)=0$. For $p \in[0, n)$, the function $\varphi(t) / \sqrt{t}=t^{\frac{p-n}{2(n+p)}}$ is strictly decreasing. Since $\varphi^{\prime \prime}(t)<0$ for $p \in(0, n)$, this shows that $\varphi \in \operatorname{Conc}^{-}(0, \infty)$ for all $p \in(0, n)$. Moreover, $\varphi$ is strictly increasing when $p \in(0, n]$, and $\varphi(t) \varphi(1 / t)=\varphi(1)^{2}$. Thus $\varphi$ satisfies the hypotheses of Theorem 6.4 for any $p \in(0, n)$, so $\operatorname{IS}_{p}(K) \operatorname{IS}_{p}\left(K^{\circ}\right) \leq \operatorname{IS}_{p}\left(B_{n}\right)^{2}$ holds for all $p \in(0, n)$.

For $p=0$, it was shown in [18] that $\operatorname{IS}_{0}(K)=n|K|$. Thus by the Blaschke-Santaló inequality,

$$
\mathrm{IS}_{0}(K) \mathrm{IS}_{0}\left(K^{\circ}\right)=n^{2}|K| \cdot\left|K^{\circ}\right| \leq n^{2}\left|B_{n}\right|^{2}=\mathrm{IS}_{0}\left(B_{n}\right)^{2} .
$$

For $p=n$, it was also shown in [18] that $\operatorname{IS}_{n}(K)=\left|\partial B_{n}\right|$, so

$$
\operatorname{IS}_{n}(K) \operatorname{IS}_{n}\left(K^{\circ}\right)=\left|\partial B_{n}\right|^{2}=\operatorname{IS}_{n}\left(B_{n}\right)^{2}
$$

and equality holds trivially. This proves (i). 
For (ii), note that $\varphi^{*}(t)=t^{\frac{n}{n+p}}$. From (17) and (39) it follows that (see also [25], [61, Cor. 3.1] and [34, Thm. 4])

$$
\operatorname{as}_{\varphi^{*}}(K)=\operatorname{as}_{n^{2} / p}(K)
$$

Therefore, by (17), the Blaschke-Santaló inequality for $\mathrm{IS}_{p}$ and Lemma 5.1 ,

$$
\mathrm{OS}_{n^{2} / p}(K) \operatorname{OS}_{n^{2} / p}\left(K^{\circ}\right)=\operatorname{IS}_{p}(K) \operatorname{IS}_{p}\left(K^{\circ}\right) \leq \operatorname{IS}_{p}\left(B_{n}\right)^{2}=\mathrm{OS}_{n^{2} / p}\left(B_{n}\right)^{2}
$$

Now we replace $n^{2} / p$ for $p \in(0, n]$ by $p$ for $p \in[n, \infty)$ to obtain the desired inequality. In the case $p=n$, equality holds trivially since

$$
\mathrm{OS}_{n}(K) \mathrm{OS}_{n}\left(K^{\circ}\right)=\operatorname{IS}_{n}(K) \operatorname{IS}_{n}\left(K^{\circ}\right)=\mathrm{IS}_{n}\left(B_{n}\right)^{2}
$$

For the special case $p=\infty$, we apply (43) and the Blaschke-Santaló inequality to get

$$
\begin{aligned}
\operatorname{OS}_{\infty}(K) \mathrm{OS}_{\infty}\left(K^{\circ}\right) & =\sup _{K^{\prime} \in \mathscr{C}_{\text {out }}(K)} \operatorname{as}_{\infty}\left(K^{\prime}\right) \cdot \sup _{K^{\prime \prime} \in \mathscr{C}_{\text {out }}\left(K^{\circ}\right)} \operatorname{as}_{\infty}\left(K^{\prime \prime}\right) \\
& \leq \sup _{K^{\prime} \in \mathscr{C}_{\text {out }}(K)} n\left|\left(K^{\prime}\right)^{\circ}\right| \cdot \sup _{K^{\prime \prime} \in \mathscr{C}_{\text {out }}\left(K^{\circ}\right)} n\left|\left(K^{\prime \prime}\right)^{\circ}\right| \\
& =\sup _{\left(K^{\prime}\right)^{\circ} \in \mathscr{C}_{\text {in }}\left(K^{\circ}\right)} n\left|\left(K^{\prime}\right)^{\circ}\right| \cdot \sup _{\left(K^{\prime \prime}\right)^{\circ} \in \mathscr{C}_{\text {in }}(K)} n\left|\left(K^{\prime \prime}\right)^{\circ}\right| \\
& \leq n^{2}|K| \cdot\left|K^{\circ}\right| \\
& \leq n^{2}\left|B_{n}\right|^{2} \\
& =\mathrm{OS}_{\infty}\left(B_{n}\right)^{2}
\end{aligned}
$$

The equality conditions in parts (i) and (ii) follow from those in Theorem 6.4.

We also obtain the following inverse Santaló type inequalities for the extremal $L_{p}$ affine surface areas. To state the result, we first define the following notation. For $p<-n$ and $\psi(t)=$ $t^{\frac{n}{n+p}}$, let $\operatorname{as}_{p}^{*}(K):=\operatorname{as}_{\psi}^{*}(K)=\operatorname{as}_{\psi}\left(K^{\circ}\right)=\operatorname{as}_{n^{2} / p}\left(K^{\circ}\right)$ and define is ${ }_{p}^{*}(K):=\inf _{K^{\prime} \in \mathscr{C}_{\text {in }}(K)} \operatorname{as}_{p}^{*}\left(K^{\prime}\right)$.

Theorem 6.8. Let $K$ be a convex body in $\mathbb{R}^{n}$ with centroid at the origin.

(i) Let $p \in(-n, 0]$. There exists a positive absolute constant c such that $\operatorname{os}_{p}(K) \operatorname{os}_{p}\left(K^{\circ}\right) \geq$ $c^{\frac{n(n-p)}{n+p}} \operatorname{OS}_{p}\left(B_{n}\right)^{2}$.

(ii) Let $p \in(-\infty,-n)$. There exists a positive absolute constant c such that $\operatorname{is}_{p}^{*}(K)$ is $_{p}^{*}\left(K^{\circ}\right) \geq$ $c^{\frac{n(n-p)}{n+p}} \operatorname{is}_{p}^{*}\left(B_{n}\right)^{2}$. 
Proof. Using inequalities (42) and (36) we derive

$$
\begin{aligned}
\operatorname{os}_{p}(K) \operatorname{os}_{p}\left(K^{\circ}\right) & =\inf _{K \in \mathscr{C}_{\text {out }}(K)} \operatorname{as}_{p}\left(K^{\prime}\right) \cdot \inf _{K^{\prime \prime} \in \mathscr{C}_{\text {out }}\left(K^{\circ}\right)} \operatorname{as}_{p}\left(K^{\prime \prime}\right) \\
& \geq \inf _{K^{\prime} \in \mathscr{C}_{\text {out }}(K)} \operatorname{vrad}\left(K^{\prime}\right)^{\frac{n(n-p)}{n+p}} \operatorname{as}_{p}\left(B_{n}\right) \cdot \inf _{K^{\prime \prime} \in \mathscr{C}_{\text {out }}\left(K^{\circ}\right)} \operatorname{vrad}\left(K^{\prime \prime}\right)^{\frac{n(n-p)}{n+p}} \operatorname{as}_{p}\left(B_{n}\right) \\
& =\left(\operatorname{vrad}(K) \operatorname{vrad}\left(K^{\circ}\right)\right)^{\frac{n(n-p)}{n+p}} \operatorname{as}_{p}\left(B_{n}\right)^{2} \\
& \geq c^{\frac{n(n-p)}{n+p}} \operatorname{as}_{p}\left(B_{n}\right)^{2} \\
& =c^{\frac{n(n-p)}{n+p}} \operatorname{os}_{p}\left(B_{n}\right)^{2} .
\end{aligned}
$$

Part (ii) now follows from (i) by using is ${ }_{p}^{*}(K)=\mathrm{os}_{n^{2} / p}\left(K^{\circ}\right)$ for $p \in(-\infty,-n)$.

\section{Appendix}

7.1. Proof of Lemma 4.1. The proof is similar to that of [18, Lemma 3.2]; we include the arguments for the reader's convenience. Let $\varphi \in \operatorname{Conc}^{-}(0, \infty)$. By Lemmas 2.1 and 3.2,

$$
\begin{aligned}
\operatorname{IS}_{\varphi}(K)=\sup _{K^{\prime} \in \mathscr{C}_{\text {in }}(K)} \operatorname{as}_{\varphi}\left(K^{\prime}\right) & \leq \sup _{K^{\prime} \in \mathscr{C}_{\text {in }}(K)} \operatorname{as}_{\varphi}\left(\operatorname{vrad}\left(K^{\prime}\right) B_{n}\right) \\
& =\sup _{K^{\prime} \in \mathscr{C}_{\text {in }}(K)} \operatorname{vrad}\left(K^{\prime}\right)^{n} \varphi\left(\operatorname{vrad}\left(K^{\prime}\right)^{-2 n}\right)\left|\partial B_{n}\right| \\
& \leq \operatorname{vrad}(K)^{n} \varphi\left(\operatorname{vrad}(K)^{-2 n}\right)\left|\partial B_{n}\right| \\
& =n|K| \varphi\left(\operatorname{vrad}(K)^{-2 n}\right),
\end{aligned}
$$

which is finite. Hence, there exists a sequence $\left\{C_{k}\right\}_{k \in \mathbb{N}} \subset \mathscr{C}_{\text {in }}(K)$ such that for all $k \in \mathbb{N}$,

$$
\operatorname{as}_{\varphi}\left(C_{k}\right)+\frac{1}{k} \geq \sup _{K^{\prime} \in \mathscr{C}_{\text {in }}(K)} \operatorname{as}_{\varphi}\left(K^{\prime}\right) .
$$

On the other hand, $\operatorname{as}_{\varphi}\left(C_{k}\right) \leq \sup _{K^{\prime} \in \mathscr{C}_{\text {in }}(K)} \operatorname{as}_{\varphi}\left(K^{\prime}\right)$ since $C_{k} \in \mathscr{C}_{\text {in }}(K)$, so by the squeeze theorem

$$
\lim _{k \rightarrow \infty} \operatorname{as}_{\varphi}\left(C_{k}\right)=\sup _{K^{\prime} \in \mathscr{C}_{\text {in }}(K)} \operatorname{as}_{\varphi}\left(K^{\prime}\right) .
$$

By the Blaschke selection theorem, there exists a subsequence $\left\{C_{k_{j}}\right\}_{j \in \mathbb{N}}$ that converges to a convex set $K_{0} \subset K$ with respect to the Hausdorff metric as $j \rightarrow \infty$. We claim that $K_{0}$ has nonempty interior. To see this, suppose not. Then $\lim _{j \rightarrow \infty}\left|C_{k_{j}}\right|=\left|K_{0}\right|=0$. By Lemma 2.1 and the definition of $\operatorname{Conc}^{-}(0, \infty)$ this implies

$$
\lim _{j \rightarrow \infty} \operatorname{as}_{\varphi}\left(C_{k_{j}}\right) \leq \lim _{j \rightarrow \infty} \operatorname{as}_{\varphi}\left(\operatorname{vrad}\left(C_{k_{j}}\right) B_{n}\right)=\lim _{j \rightarrow \infty} \operatorname{vrad}\left(C_{k_{j}}\right)^{n} \varphi\left(\operatorname{vrad}\left(C_{k_{j}}\right)^{-2 n}\right)\left|\partial B_{n}\right|=0 .
$$

Since $K$ contains the origin in its interior, there exists $\varepsilon>0$ such that $\varepsilon B_{n} \subset K$. Thus,

$$
0=\lim _{j \rightarrow \infty} \operatorname{as}_{\varphi}\left(C_{k_{j}}\right)=\sup _{K^{\prime} \in \mathscr{C}_{\text {in }}(K)} \operatorname{as}_{\varphi}\left(K^{\prime}\right) \geq \operatorname{as}_{\varphi}\left(\varepsilon B_{n}\right)=\varepsilon^{n} \varphi\left(\varepsilon^{-2 n}\right)\left|\partial B_{n}\right|>0,
$$

a contradiction. We have therefore shown that $K_{0}$ is a convex body in $\mathbb{R}^{n}$. 
Finally, we show that $\operatorname{IS}_{\varphi}(K)=\operatorname{as}_{\varphi}\left(K_{0}\right)$. On one hand, $\operatorname{IS}_{\varphi}(K) \geq \operatorname{as}_{\varphi}\left(K_{0}\right)$ since $K_{0} \in$ $\mathscr{C}_{\text {in }}(K)$, while on the other hand we can apply the upper semicontinuity (9) of the $L_{\varphi}$ affine surface area to get

$$
\operatorname{IS}_{\varphi}(K)=\sup _{K^{\prime} \in \mathscr{C}_{\text {in }}(K)} \operatorname{as}_{\varphi}\left(K^{\prime}\right)=\limsup _{j \rightarrow \infty} \operatorname{as}_{\varphi}\left(C_{k_{j}}\right) \leq \operatorname{as}_{\varphi}\left(K_{0}\right) .
$$

This proves (i).

Next, we show (iii), again following the arguments in [18]. The proof is similar to that of (i), but to apply the Blaschke selection theorem we first have to show that any body achieving the infimum lies inside of a larger body that we can draw a convergent subsequence from. Since $K$ is bounded, there exists $R>0$ such that $K \subset R B_{n}$. First, we show that for all $K^{\prime} \in \mathscr{C}_{\text {out }}(K)$ with $\left|K^{\prime}\right| \geq(R n)^{n}\left|B_{n}\right|$, there exists $\tilde{K} \in \mathscr{C}_{\text {out }}(K)$ such that $\tilde{K} \subset R n B_{n}$ and $\operatorname{as}_{\psi}\left(K^{\prime}\right) \geq \operatorname{as}_{\psi}(\tilde{K})$. There exists an affine transformation $A: \mathbb{R}^{n} \rightarrow \mathbb{R}^{n}$ with $|\operatorname{det}(A)|=1$ and a positive number $t$ such that $t B_{n}$ has maximum volume among all ellipsoids contained in $A\left(K^{\prime}\right)$. By F. John's theorem [27,

$$
t B_{n} \subset A\left(K^{\prime}\right) \subset \operatorname{tn} B_{n} .
$$

Thus

$$
(R n)^{n}\left|B_{n}\right| \leq\left|K^{\prime}\right|=\left|A\left(K^{\prime}\right)\right| \leq(t n)^{n}\left|B_{n}\right|,
$$

which implies $R \leq t$. Hence,

$$
K \subset R B_{n}=\frac{R}{t} \cdot t B_{n} \subset \frac{R}{t} A\left(K^{\prime}\right) .
$$

Now consider the affine transformation $T=r A$ with $r=R / t \leq 1$, and set $\tilde{K}:=T\left(K^{\prime}\right)$. Then by (44), $\tilde{K} \subset \frac{R}{t}\left(t n B_{n}\right)=R n B_{n}$ and $\tilde{K} \supset \frac{R}{t}\left(t B_{n}\right)=R B_{n} \supset K$. By Lemma [5.2, $\operatorname{as}_{\psi}\left(r K^{\prime}\right) \leq r^{n} \operatorname{as}_{\psi}\left(K^{\prime}\right)$. The SL( $\left.n\right)$ invariance of $\operatorname{as}_{\psi}$ means that $\operatorname{as}_{\psi}(A(K))=\operatorname{as}_{\psi}(K)$ for any affine transformation $A$ with $|\operatorname{det}(A)|=1$. Putting all of this together, we obtain

$$
\operatorname{as}_{\psi}(\tilde{K})=\operatorname{as}_{\psi}\left(r A\left(K^{\prime}\right)\right) \leq r^{n} \operatorname{as}_{\psi}\left(A\left(K^{\prime}\right)\right)=r^{n} \operatorname{as}_{\psi}\left(K^{\prime}\right) \leq \operatorname{as}_{\psi}\left(K^{\prime}\right) .
$$

This proves the claim regarding those $K^{\prime} \in \mathscr{C}_{\text {out }}(K)$ with $\left|K^{\prime}\right| \geq(R n)^{n}\left|B_{n}\right|$.

Now suppose that $K^{\prime} \in \mathscr{C}_{\text {out }}(K)$ satisfies $\left|K^{\prime}\right| \leq(R n)^{n}\left|B_{n}\right|$. Since $K^{\prime}$ has nonempty interior, there exists $r>0$ such that $r B_{n} \subset K^{\prime}$. For each $x \in K^{\prime}$, consider the cone $C_{x}$ with apex $x$ and base $x^{\perp} \cap r B_{n}$, and let $h_{x}$ denote the height of this cone. Then $C_{x} \subset K^{\prime}$, so

$$
\left|C_{x}\right|=\frac{h_{x} r^{n-1}}{n}\left|B_{n-1}\right| \leq\left|K^{\prime}\right| \leq(R n)^{n}\left|B_{n}\right|
$$

which implies $K^{\prime} \subset \frac{n^{n+1} R^{n}\left|B_{n}\right|}{r^{n-1}\left|B_{n-1}\right|} B_{n}$.

Therefore, in these two cases we have shown that any convex body $K^{\prime} \in \mathscr{C}_{\text {out }}(K)$ that achieves the infimum must be contained in the ball $\max \left\{R n, \frac{n^{n+1} R^{n}\left|B_{n}\right|}{r^{n-1}\left|B_{n-1}\right|}\right\} B_{n}$. So assume that 
$K^{\prime}$ is contained in this ball. We can now use the argument from the proof of (i). By Lemma 2.2. (12) and (13) we derive

$$
\operatorname{as}_{\psi}\left(K^{\prime}\right) \geq \operatorname{as}_{\psi}\left(\operatorname{vrad}\left(K^{\prime}\right) B_{n}\right) \geq \operatorname{as}_{\psi}\left(\operatorname{vrad}(K) B_{n}\right) .
$$

This shows that the infimum $\operatorname{os}_{\psi}(K)$ is finite. Now as before, there exists a sequence $\left\{C_{k}\right\}_{k \in \mathbb{N}}$ of convex bodies in $\mathscr{C}_{\text {out }}(K)$ such that for all $k \in \mathbb{N}$,

$$
\operatorname{as}_{\psi}\left(C_{k}\right) \leq \inf _{K^{\prime} \in \mathscr{C}_{\text {out }}(K)} \operatorname{as}_{\psi}\left(K^{\prime}\right)+\frac{1}{k} .
$$

Conversely, $\operatorname{as}_{\psi}\left(C_{k}\right) \geq \inf _{K^{\prime} \in \mathscr{C}_{\text {out }}(K)} \operatorname{as}_{\psi}\left(K^{\prime}\right)$ since $C_{k} \in \mathscr{C}_{\text {out }}(K)$, so by another application of the squeeze theorem we deduce that

$$
\lim _{k \rightarrow \infty} \operatorname{as}_{\psi}\left(C_{k}\right)=\operatorname{os}_{\psi}(K)
$$

By the Blaschke selection theorem, there exists a subsequence $\left\{C_{k_{j}}\right\}_{j \in \mathbb{N}}$ in $\mathscr{C}_{\text {out }}(K)$ that converges to a convex set $K_{0} \supset K$ with respect to the Hausdorff metric as $j \rightarrow \infty$. Since $K_{0} \supset K$, it has nonempty interior. Therefore, we may apply the lower semicontinuity (10) of $\operatorname{as}_{\psi}$ to derive

$$
\operatorname{os}_{\psi}(K)=\inf _{K \in \mathscr{C}_{\text {out }}(K)} \operatorname{as}_{\psi}\left(K^{\prime}\right)=\liminf _{j \rightarrow \infty} \operatorname{as}_{\psi}\left(C_{k_{j}}\right) \geq \operatorname{as}_{\psi}\left(K_{0}\right) .
$$

Part (ii) now follows from (i) and the formula $\mathrm{OS}_{\varphi^{*}}(K)=\operatorname{IS}_{\varphi}(K)$, and (iv) follows from (iii) and the formula is ${ }_{\psi}^{*}(K)=\operatorname{os}_{\psi}\left(K^{\circ}\right)$.

7.2. Proof of Proposition 4.2. The argument is similar to that of [18, Prop. 3.3]. By hypothesis, $K$ has centroid at the origin, so there exists a Euclidean ball $\rho B_{n}$ of positive radius $\rho>0$ that is contained in $K$, and a sequence of convex bodies $\left\{K_{\ell}\right\}_{\ell \in \mathbb{N}}$ that have centroid at the origin and converge to $K$ in the Hausdorff metric. Thus, for every $\varepsilon>0$ there exists an integer $\ell_{0}$ such that for all $\ell \geq \ell_{0}$,

$$
K_{\ell} \subset K+\varepsilon B_{n} \quad \text { and } \quad K \subset K_{\ell}+\varepsilon B_{n} .
$$

For sufficiently small $\varepsilon$, we may assume that for all $\ell \geq \ell_{0}$, the inclusion $\frac{\rho}{10} B_{n} \subset K_{\ell}$ holds. The previous inclusions together imply that for all $\ell \geq \ell_{0}$,

$$
K_{\ell} \subset K+\varepsilon B_{n}=K+\frac{\varepsilon}{\rho} \cdot \rho B_{n} \subset K+\frac{\varepsilon}{\rho} K=\left(1+\frac{\varepsilon}{\rho}\right) K
$$

and

$$
K \subset K_{\ell}+\varepsilon B_{n}=K_{\ell}+\frac{10 \varepsilon}{\rho} \cdot \frac{\rho}{10} B_{n} \subset K_{\ell}+\frac{10 \varepsilon}{\rho} K_{\ell}=\left(1+\frac{10 \varepsilon}{\rho}\right) K_{\ell} .
$$

From (45), the monotonicity property (19) of $\mathrm{IS}_{\varphi}$ and Lemma [5.2, we get that for all $\ell \geq \ell_{0}$,

$$
\operatorname{IS}_{\varphi}\left(K_{\ell}\right) \leq \operatorname{IS}_{\varphi}\left(\left(1+\frac{\varepsilon}{\rho}\right) K\right)=\sup _{K^{\prime} \in \mathscr{C}_{\text {in }}(K)} \operatorname{as}_{\varphi}\left(\left(1+\frac{\varepsilon}{\rho}\right) K^{\prime}\right) \leq\left(1+\frac{\varepsilon}{\rho}\right)^{n} \operatorname{IS}_{\varphi}(K)
$$


Similarly, from (46) we derive that for all $\ell \geq \ell_{0}$,

$$
\operatorname{IS}_{\varphi}(K) \leq\left(1+\frac{10 \varepsilon}{\rho}\right)^{n} \operatorname{IS}_{\varphi}\left(K_{\ell}\right) .
$$

The previous two inequalities now imply that for all $\ell \geq \ell_{0}$,

$$
\left(1+\frac{\varepsilon}{\rho}\right)^{-n} \operatorname{IS}_{\varphi}\left(K_{\ell}\right) \leq \operatorname{IS}_{\varphi}(K) \leq\left(1+\frac{10 \varepsilon}{\rho}\right)^{n} \operatorname{IS}_{\varphi}\left(K_{\ell}\right) .
$$

Since $\varepsilon$ was arbitrary, claim (i) follows.

Next, let $\varphi^{*} \in \operatorname{Conc}^{+}(0, \infty)$. Then for all $\ell \geq \ell_{0}$, inclusions (47) and (48) imply that $K_{\ell}^{\circ} \supset\left(1+\frac{\varepsilon}{\rho}\right)^{-1} K^{\circ}$ and $K^{\circ} \supset\left(1+\frac{10 \varepsilon}{\rho}\right)^{-1} K_{\ell}^{\circ}$, respectively. Thus for all $\ell \geq \ell_{0}$,

$$
\operatorname{OS}_{\varphi^{*}}\left(K_{\ell}\right)=\operatorname{IS}_{\varphi}\left(K_{\ell}^{\circ}\right) \geq \operatorname{IS}_{\varphi}\left(\left(1+\frac{\varepsilon}{\rho}\right)^{-1} K^{\circ}\right) \geq\left(1+\frac{\varepsilon}{\rho}\right)^{-n} \operatorname{IS}_{\varphi}\left(K^{\circ}\right)=\left(1+\frac{\varepsilon}{\rho}\right)^{-n} \operatorname{OS}_{\varphi^{*}}(K)
$$

and

$$
\begin{aligned}
\operatorname{OS}_{\varphi^{*}}(K)=\operatorname{IS}_{\varphi}\left(K^{\circ}\right) & \geq \operatorname{IS}_{\varphi}\left(\left(1+\frac{10 \varepsilon}{\rho}\right)^{-1} K_{\ell}^{\circ}\right) \\
& \geq\left(1+\frac{10 \varepsilon}{\rho}\right)^{-n} \operatorname{IS}_{\varphi}\left(K_{\ell}^{\circ}\right)=\left(1+\frac{10 \varepsilon}{\rho}\right)^{-n} \operatorname{OS}_{\varphi^{*}}\left(K_{\ell}\right) .
\end{aligned}
$$

Therefore, for all $\ell \geq \ell_{0}$ we have

$$
\left(1+\frac{10 \varepsilon}{\rho}\right)^{-n} \operatorname{OS}_{\varphi^{*}}\left(K_{\ell}\right) \leq \operatorname{OS}_{\varphi^{*}}(K) \leq\left(1+\frac{\varepsilon}{\rho}\right)^{n} \operatorname{OS}_{\varphi^{*}}\left(K_{\ell}\right) .
$$

Since $\varepsilon>0$ was arbitrary, claim (ii) follows. The arguments to prove (iii) and (iv) are similar.

7.3. Proof of Remark 3.3. For any $t>0$, we have $\varphi_{m}(t)>0$. The first two derivatives of $\varphi_{m}$ are:

$$
\begin{aligned}
\varphi_{m}^{\prime}(t) & =\frac{1}{2 m}\left(t^{\frac{m-1}{m}}+t^{\frac{m+1}{m}}\right)^{-1} \\
\varphi_{m}^{\prime \prime}(t) & =-\frac{1}{m^{2}}\left(t^{\frac{m-1}{m}}+t^{\frac{m+1}{m}}\right)^{-2}\left((m-1) t^{-\frac{1}{m}}+(m+1) t^{\frac{1}{m}}\right) .
\end{aligned}
$$

Since $\varphi_{m}^{\prime \prime}(t)<0$ for all $t>0$, the function $\varphi_{m}:(0, \infty) \rightarrow(0, \infty)$ is concave. By continuity, $\lim _{t \rightarrow 0} \varphi_{m}(t)=\arctan (0)=0$ and since $\varphi_{m}(t)$ is monotonically increasing and bounded above by $\pi / 2$,

$$
0 \leq \lim _{t \rightarrow \infty} \frac{\varphi_{m}(t)}{t} \leq \lim _{t \rightarrow \infty} \frac{\pi}{2 t}=0 .
$$

Furthermore, $\varphi_{m}(0)=0$, which shows that $\varphi_{m} \in \operatorname{Conc}(0, \infty)$. 
To prove that $\varphi_{m} \in \operatorname{Conc}^{-}(0, \infty)$, it remains to show that $\varphi_{m}(t) / \sqrt{t}$ is decreasing. We have $\frac{d}{d t}\left(\varphi_{m}(t) / \sqrt{t}\right)<0$ if and only if $\varphi_{m}^{\prime}(t)<\frac{\varphi_{m}(t)}{2 t}$, which is equivalent to

$$
t^{1 / m}+t^{-1 / m}>\left[\frac{m}{2} \cdot \arctan \left(t^{1 / m}\right)\right]^{-1} \text {. }
$$

We show that $(\arctan (x))^{-1}<x+\frac{1}{x}$ for any $x>0$, which is equivalent to $\arctan (x)>\frac{x}{x^{2}+1}$ for $x>0$. Let $g(x):=\arctan (x)-\frac{x}{x^{2}+1}$. Then $g^{\prime}(x)=\frac{2 x^{2}}{\left(x^{2}+1\right)^{2}}>0$ for any $x>0$, so $g$ is increasing on $(0, \infty)$. Thus for any $x>0$ we have $g(x)>g(0)=0$, which proves the inequality. Taking $x=t^{1 / m}$ and using the fact that $m \geq 2$, we derive

$$
\left[\frac{m}{2} \cdot \arctan \left(t^{1 / m}\right)\right]^{-1}=\frac{2}{m}\left(\arctan \left(t^{1 / m}\right)\right)^{-1} \leq\left(\arctan \left(t^{1 / m}\right)\right)^{-1}<t^{1 / m}+t^{-1 / m} .
$$

7.4. Remarks on the Centroid Assumption. We defined $\mathscr{C}_{\text {in }}(K)$ and $\mathscr{C}_{\text {out }}(K)$ to include only bodies with centroid at the origin so that we can apply the $L_{\varphi}$ and $L_{\psi}$ affine isoperimetric inequalities in Lemmas 2.1 and 2.2, respectively. This, in turn, allows us to state our results for the broad range of functions in $\operatorname{Conc}^{-}(0, \infty) \cup \operatorname{Conc}^{+}(0, \infty)$ and all of the functions in $\operatorname{Conv}(0, \infty)$.

Ye [65] used Steiner symmetrizations to prove the following $L_{\varphi}$ and $L_{\psi}$ affine isoperimetric inequalities. These inequalities do not have the restriction that the centroid of the body is the origin; on the other hand, they impose some additional restrictions on the functions $\varphi$ and $\psi$, and in the former case, on the curvature of $K$.

Lemma 7.1. 65, Thm. 3.2] Let $K \in \mathcal{K}_{o}^{n}$ and suppose that $\varphi \in \operatorname{Conc}(0, \infty)$ is such that the function $F_{n}(t)=\varphi\left(t^{n+1}\right)$ for $t \in(0, \infty)$ is concave. Then

$$
\operatorname{as}_{\varphi}(K) \leq \operatorname{as}_{\varphi}\left(\operatorname{vrad}(K) B_{n}\right) .
$$

Moreover, if $F_{n}(\cdot)$ is strictly concave and $K$ has positive Gaussian curvature almost everywhere, then equality holds if and only if $K$ is an origin-symmetric ellipsoid.

Lemma 7.2. 65, Thm. 3.4] Let $K \in \mathcal{K}_{o}^{n}$ and suppose that $\psi \in \operatorname{Conc}(0, \infty)$ is such that the function $G_{n}(t)=\psi\left(t^{n+1}\right)$ for $t \in(0, \infty)$ is convex. Then

$$
\operatorname{as}_{\psi}(K) \geq \operatorname{as}_{\psi}\left(\operatorname{vrad}(K) B_{n}\right) .
$$

Moreover, if $G_{n}(\cdot)$ is strictly convex, then equality holds if and only if $K$ is an origin-symmetric ellipsoid.

Thus, one could remove the assumption that the bodies in $\mathscr{C}_{\text {in }}(K)$ or $\mathscr{C}_{\text {out }}(K)$ have centroid at the origin by using Lemmas 7.1 and 7.2 in all of the proofs instead of Lemmas 2.1 and 2.2. but the downside is then one captures fewer functions from $\operatorname{Conc}(0, \infty)$ and $\operatorname{Conv}(0, \infty)$. Furthermore, the assumption in the definitions of $\mathscr{C}_{\text {in }}(K)$ and $\mathscr{C}_{\text {out }}(K)$ that the bodies have centroid at the origin is tacitly assumed in the definition of the extremal $L_{p}$ affine surface areas in [18]. This is because the definition (39) of $L_{p}$ affine surface area is stated for convex bodies with centroid at the origin. Ultimately, it is an open problem to obtain the results of this article for a richer class of functions than $\operatorname{Conc}^{-}(0, \infty) \cup \operatorname{Conc}^{+}(0, \infty)$. 
STEVEN HOEHNER

\section{ACKNOWLEDGMENTS}

The author would like to thank Elisabeth Werner and Deping Ye for the discussions, and the anonymous referee for carefully reading this manuscript and providing valuable comments.

\section{REFERENCES}

[1] S. Artstein-Avidan, A. Giannopoulos, and V. D. Milman. Asymptotic Geometric Analysis, Part I. volume 202 of Mathematical Surveys and Monographs. American Mathematical Society, 2015.

[2] K. Ball. Volume ratios and a reverse isoperimetric inequality. Journal of the London Mathematical Society, 44(2):351-359, 1991.

[3] I. Bárány. The Limit Shape of Convex Lattice Polygons. Discrete 83 Computational Geometry, 13:279-295, 1995.

[4] I. Bárány. Affine perimeter and limit shape. Journal für die reine und angewandte Mathematik, 484:71-84, 1997.

[5] I. Bárány. Sylvester's question: the probability that $n$ points are in convex position. Annals of Probability, 27:2020-2034, 1999.

[6] I. Bárány and M. Prodromou. On maximal convex lattice polygons inscribed in a plane convex set. Israel Journal of Mathematics, 154:337-360, 2006.

[7] F. Barthe. An extremal property of the mean width of the simplex. Mathematische Annalen, 310:685-693, 1998.

[8] W. Blaschke. Über affine Geometrie VII: Neue Extremeingenschaften von Ellipse und Ellipsoid. Ber. Verh. Sächs. Akad. Wiss., Math. Phys. Kl., 69:412-420, 1917.

[9] W. Blaschke. Vorlesunger über Differentialgeometrie: Affine Differentialgeometrie. Springer Verlag, Berlin, 1923.

[10] J. Bourgain and V. D. Milman. New volume ratio properties for convex symmetric bodies in $\mathbb{R}^{n}$. Inventiones mathematicae, 88:319-340, 1987.

[11] S. Brazitikos, A. Giannopoulos, P. Valettas, and B.-H. Vritsiou. Geometry of isotropic convex bodies. volume 196 of Mathematical Surveys and Monographs. American Mathematical Society, 2014.

[12] U. Caglar and D. Ye. Affine isoperimetric inequalities in the functional Orlicz-Brunn-Minkowski theory. Advances in Applied Mathematics, 81:78-114, 2016.

[13] F. Chen, J. Zhou, and C. Yang. On the reverse Orlicz Busemann-Petty centroid inequality. Advances in Applied Mathematics, 47:820-828, 2011.

[14] L. Davies. The Asymptotics of Rousseeuw's Minimum Volume Ellipsoid Estimator. Annals of Statistics, 20(4), 1992.

[15] T. Figiel, J. Lindenstrauss, and V. D. Milman. The dimension of almost spherical sections of convex bodies. Acta Mathematica, 139:53-94, 1977.

[16] R. J. Gardner, D. Hug, S. Xing, and D. Ye. General volumes in the Orlicz-Brunn-Minkowski theory and a related Minkowski problem II. Calculus of Variations and Partial Differential Equations, 59(1):Article no. $15,2020$.

[17] A. A. Giannopoulos and V. D. Milman. Extremal problems and isotropic positions of convex bodies. Israel Journal of Mathematics, 117:29-60, 2000.

[18] O. Giladi, H. Huang, C. Schütt, and E. Werner. Constrained convex bodies with extremal affine surface areas. Journal of Functional Analysis, 279(3):Article 108531, 2020.

[19] M. Grötschel, L. Lovász, and A. Schrijver. Geometric algorithms and combinatorial optimization. Algorithms and Combinatorics. Springer, 2 edition, 1993.

[20] P. M. Gruber. Asymptotic estimates for best and stepwise approximation of convex bodies II. Forum Math., 5:521-538, 1993. 
[21] O. Guédon and E. Milman. Interpolating thin-shell and sharp large-deviation estimates for isotropic log-concave measures. Geom. Funct. Anal., 21:1043-1068, 2011.

[22] C. Haberl, E. Lutwak, D. Yang, and G. Zhang. The even Orlicz Minkowski problem. Advances in Mathematics, 224:2485-2510, 2010.

[23] Q. Huang and B. He. On the Orlicz Minkowski Problem for Polytopes. Discrete \& Computational Geometry, 48:281-297, 2012.

[24] D. Hug. Contributions to affine surface area. manuscripta mathematica, 91:283-301, 1996.

[25] D. Hug. Curvature relations and affine surface area for a general convex body and its polar. Results in Mathematics, 29:233-248, 1996.

[26] H. Jian and J. Lu. Existence of solutions to the Orlicz-Minkowski problem. Advances in Mathematics, 344:262-288, 2019.

[27] F. John. Extremum problems with inequalities as subsidiary conditions. Studies and Essays Presented to R. Courant on his 60th Birthday, pages 187-204. Interscience Publishers, Inc., New York, 1948.

[28] G. Kuperberg. From the Mahler Conjecture to Gauss Linking Integrals. Geometric and Functional Analysis, 18:870-892, 2008.

[29] K. Leichtweiß. Zur Affinoberfläche konvexer Körper. manuscripta mathematica, 91(3):429-464, 1986.

[30] K. Leichtweiß. Über einige Eigenschaften der Affinoberfläche beliebiger konvexer Körper. Results in Mathematics, 13:255-282, 1988.

[31] A. Li and G. Leng. A New Proof of the Orlicz Busemann-Petty Centroid Inequality. Proceedings of the American Mathematical Society, 139(4):1473-1481, 2011.

[32] J. Liu and B. Zhou. An obstacle problem for a class of Monge-Ampère type functionals. Journal of Differential Equations, 254:1306-1325, 2013.

[33] M. Ludwig. Asymptotic approximation of smooth convex bodies by general polytopes. Mathematika, 46:103-125, 1999.

[34] M. Ludwig. General affine surface areas. Advances in Mathematics, 224(6):2346-2360, 2010.

[35] M. Ludwig and M. Reitzner. A classification of SL $(n)$ invariant valuations. Annals of Mathematics, 172(2):1219-1267, 2010.

[36] E. Lutwak. Extended affine surface area. Advances in Mathematics, 85:39-68, 1991.

[37] E. Lutwak. The Brunn-Minkowski-Firey Theory II: Affine and Geominimal Surface Areas. Advances in Mathematics, 118:244-294, 1996.

[38] E. Lutwak, D. Yang, and G. Zhang. Orlicz centroid bodies. Journal of Differential Geometry, 84(2):365$387,2010$.

[39] E. Lutwak, D. Yang, and G. Zhang. Orlicz projection bodies. Advances in Mathematics, 223(1):220-242, 2010.

[40] M. Meyer and E. Werner. On the p-affine surface area. Advances in Mathematics, 152:288-313, 2000.

[41] V. D. Milman and A. Pajor. Entropy and Asymptotic Geometry of Non-Symmetric Convex Bodies. Advances in Mathematics, 152(2):314-335, 2000.

[42] F. Nazarov. The Hörmander Proof of the Bourgain-Milman Theorem. In B. Klartag, S. Mendelson, and V. D. Milman, editors, Geometric Aspects of Functional Analysis: Israel Seminar 2006-2010, pages 335-343. Springer Berlin Heidelberg, Berlin, Heidelberg, 2012.

[43] C. M. Petty. Affine isoperimetric problems. Annals of the New York Academy of Sciences, 440(1):113-127, 1985.

[44] G. Pisier. The volume of convex bodies and Banach space geometry. volume 94 of Cambridge Tracts in Mathematics. Cambridge University Press, 1989.

[45] P. Rousseeuw. Multivariate estimation with high breakdown point. In W. Grossmann, G. Pflug, I. Vincze, and W. Wertz, editors, Mathematical Statistics and Applications, pages 283-297. Reidel, 1985.

[46] L. A. Santaló. Un invariante afin para los cuerpos convexos del espacio des $n$ dimensiones. Portugaliae Mathematica, 8:155-161, 1949. 
[47] M. Schmuckenschläger. The distribution function of the convolution square of a convex symmetric body in $\mathbb{R}^{n}$. Israel Journal of Mathematics, 78:309-334, 1992.

[48] M. Schmuckenschläger. An extremal property of the regular simplex. In Convex Geometric Analysis, volume 34 of Math. Sci. Res. Inst. Publ., pages 199-202. Cambridge University Press, 1999.

[49] R. Schneider. Convex Bodies: The Brunn-Minkowski Theory. Encyclopedia of Mathematics and its Applications. Cambridge University Press, 2 edition, 2013.

[50] R. Schneider. Affine surface area and convex bodies of elliptic type. Period. Math. Hung., 69:120-125, 2014.

[51] C. Schütt and E. Werner. The convex floating body. Mathematica Scandinavica, 66(2):275-290, 1990.

[52] C. Schütt and E. Werner. Polytopes with vertices chosen randomly from the boundary of a convex body. In Vitali D. Milman and Gideon Schechtman, editors, Geometric Aspects of Functional Analysis: Israel Seminar 2001-2002, pages 241-422. Springer Berlin Heidelberg, Berlin, Heidelberg, 2003.

[53] C. Schütt and E. Werner. Surface bodies and p-affine surface area. Advances in Mathematics, 187(1):98$145,2004$.

[54] W. Sheng, N. S. Trudinger, and X. Wang. Enclosed convex hypersurfaces with maximal affine area. Mathematische Zeitschrift, 252:497-510, 2006.

[55] S. Szarek. On Kašin's almost Euclidean orthogonal decomposition of $\ell_{1}^{n}$. Bull. Acad. Polon. Sci., 26:691$694,1978$.

[56] M. J. Todd and E. A. Yıldırım. On Khachiyan's algorithm for the computation of minimum-volume enclosing ellipsoids. Discrete Applied Mathematics, 155(13):1731-1744, 2007.

[57] N. S. Trudinger and X. Wang. The affine plateau problem. Journal of the American Mathematical Society, 18(2):253-289, 2004.

[58] S. Van Aelst and P. Rousseeuw. Minimum volume ellipsoid. Wiley Interdisciplinary Reviews: Computational Statistics, 1(1):71-82, 2009.

[59] W. Weidong and L. Gangsong. $L_{p}$-mixed affine surface area. Journal of Mathematical Analysis and Applications, 335:341-354, 2007.

[60] E. Werner. Illumination bodies and affine surface area. Studia Math., 110:257-269, 1994.

[61] E. Werner and D. Ye. New $L_{p}$-affine isoperimetric inequalities. Advances in Mathematics, 218:762-780, 2008.

[62] E. Werner and D. Ye. Inequalities for mixed p-affine surface area. Mathematische Annalen, 347(3), 2010.

[63] D. Xi, H. Jin, and G. Leng. The Orlicz Brunn-Minkowski inequality. Advances in Mathematics, 260:350$374,2014$.

[64] D. Ye. Inequalities for general mixed affine surface areas. Journal of the London Mathematical Society, 85(1):101-120, 2012.

[65] D. Ye. On the Monotone Properties of General Affine Surface Areas under the Steiner Symmetrization. Indiana University Mathematics Journal, 63(1):1-19, 2014.

[66] D. Ye. New Orlicz affine isoperimetric inequalities. Journal of Mathematical Analysis and Applications, 427(2):905-929, 2015.

[67] G. Zhu. The Orlicz centroid inequality for star bodies. Advances in Applied Mathematics, 48:432-445, 2012.

[68] D. Zou and G. Xiong. Orlicz-John ellipsoids. Advances in Mathematics, 265:132-168, 2014.

Department of Mathematics \& Computer Science, Longwood University, 23909 E-mail address: hoehnersd@longwood.edu 